\title{
A novel RIPK4-IRF6 connection is required to prevent epithelial fusions characteristic for popliteal pterygium syndromes
}

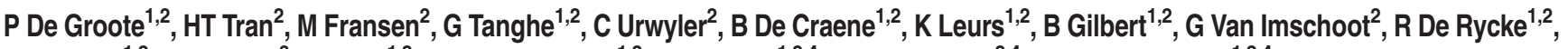 \\ CJ Guérin ${ }^{1,2}$, P Holland ${ }^{3}$, G Berx ${ }^{1,2}$, P Vandenabeele ${ }^{1,2}$, S Lippens ${ }^{1,2,4}$, K Vleminckx $^{\star, 2,4}$ and W Declercq ${ }^{\star, 1,2,4}$
}

Receptor-interacting protein kinase 4 (RIPK4)-deficient mice have epidermal defects and fusion of all external orifices. These are similar to Bartsocas-Papas syndrome and popliteal pterygium syndrome (PPS) in humans, for which causative mutations have been documented in the RIPK4 and IRF6 (interferon regulatory factor 6) gene, respectively. Although genetically distinct, these syndromes share the anomalies of marked pterygia, syndactyly, clefting and hypoplastic genitalia. Despite the strong resemblance of these two syndromes, no molecular connection between the transcription factor IRF6 and the kinase RIPK4 was known and the mechanism underlying the phenotype was unclear. Here we describe that RIPK4 deficiency in mice causes epithelial fusions associated with abnormal periderm development and aberrant ectopic localization of E-cadherin on the apical membrane of the outer peridermal cell layers. In Xenopus, RIPK4 depletion causes the absence of ectodermal epiboly and concomitant gastrulation defects that phenocopy ectopic expression of dominant-negative IRF6. We found that IRF6 controls RIPK4 expression and that wild-type, but not kinase-dead, RIPK4 can complement the gastrulation defect in Xenopus caused by IRF6 malfunctioning. In contrast to the mouse, we observed only minor effects on cadherin membrane expression in Xenopus RIPK4 morphants. However, gastrulation defects were associated with a virtual absence of cortical actin in the ectodermal cells that face the blastocoel cavity and this was phenocopied in embryos expressing dominant-negative IRF6. A role for RIPK4 in actin cytoskeleton organization was also revealed in mouse epidermis and in human epithelial HaCaT cells. In conclusion, we showed that in mice RIPK4 is implicated in cortical actin organization and in E-cadherin localization or function, which can explain the characteristic epithelial fusions observed in PPSs. In addition, we provide a novel molecular link between IRF6 and RIPK4 that unifies the different PPSs to a common molecular pathway.

Cell Death and Differentiation (2015) 22, 1012-1024; doi:10.1038/cdd.2014.191; published online 28 November 2014

The epidermis functions as a protective barrier preventing fluid loss and protecting from environmental insults. During embryogenesis, the developing epidermis is covered by a thin layer of peridermal cells. ${ }^{1}$ Upon cornification of the underlying epidermis, the periderm regresses and is shed. ${ }^{2}$ Previous studies indicated that receptor-interacting protein kinase 4 (RIPK4) has a crucial role in epidermal differentiation and can participate in cutaneous inflammation. ${ }^{3-5}$ RIPK4 is a member of the RIP serine/threonine kinase family, which act as integrators of stress signals leading to the activation of NF$\kappa \mathrm{B}$, MAP kinases, cell death or inflammation. ${ }^{6,7}$ All RIPKs contain a homologous kinase domain but have different protein-protein interaction domains allowing them to function in different pathways. RIPK4 consists of an N-terminal kinase domain, an intermediate domain and a C-terminus containing 11 ankyrin repeats.

RIPK4 ${ }^{-1-}$ mice display thickened skin showing aberrant expression of keratinocyte differentiation markers. ${ }^{3}$ However, the etiology of this phenotype remains unclear. As the outermost layer of the RIPK4 ${ }^{-1-}$ epidermis is composed of flattened nucleated cells, it was presumed that the skin of these mice is parakeratotic. RIPK4 ${ }^{-1-}$ mice also exhibit partial fusion of the limbs to the body, reduced skin folds and fusion of external orifices, including the nose, mouth and anus. ${ }^{3}$ These mice die at birth because of their inability to breathe. RIPK4 was found to be mutated in humans affected by an autosomal-recessive form of popliteal pterygium syndrome (PPS), also known as Bartsocas-Papas syndrome (BPS). ${ }^{8,9}$ Similar to RIPK4 ${ }^{-1-}$ mice, BPS patients show epithelial fusions, skin webs at flexural surfaces, atresia of the oral cavity, syndactily and alopecia, and they die perinatally. Similar to RIPK4 ${ }^{-/-}$mice, transcription factor IRF6 ${ }^{-/-}$mice display fusion of the esophagus and defective epidermal differentiation. ${ }^{10,11}$ In addition, IRF6 (interferon regulatory factor 6) and RIPK4 have been implicated in PPS. ${ }^{8,9,12}$ We found that similar to IRF6 mutants, ${ }^{10,11,13}$ RIPK4 $^{-/-}$mice

${ }^{1}$ Inflammation Research Center, Ghent 9052, Belgium; ${ }^{2}$ Department of Biomedical Molecular Biology, Ghent University, Ghent 9052, Belgium and ${ }^{3}$ Amgen, Seattle, WA 1201, USA

${ }^{*}$ Corresponding authors: K Vleminckx or W Declercq, Department for Molecular Biomedical Research, VIB, University Ghent, TechnologiePark 927, Ghent 9052, Belgium. Tel: +32 93313 721; Fax: +32 9221 7673; E-mail: Kris.Vleminckx@UGent.be or Wim.Declercq@irc.VIB-UGent.be

${ }^{4}$ These authors shared senior authorship.

Abbreviations: BCR, blastocoel roof; BPS, Bartsocas-Papas syndrome; DBD, DNA-binding domain; F-actin, filamentous-actin; Grhl3, Grainyhead-like 3; h, human; IRF6, interferon regulatory factor; IRF6DBD, IRF6 DNA-binding domain; PPS, popliteal pterygium syndrome; RIPK4, receptor-interacting protein kinase 4

Received 31.5.13; revised 21.10.14; accepted 22.10.14; Edited by D Aberdam; published online 28.11.14 
have a defective skin barrier function and cleft palate, and $\mathrm{RIPK}^{-1-}$ keratinocytes fail to differentiate in vitro. In Xenopus, we show that IRF6 controls RIPK4 expression and that RIPK4 can rescue developmental defects imposed by IRF6 dysfunction, thereby identifying a novel genetic interaction between IRF6 and RIPK4. Importantly, we found that RIPK4-deficient mice display peridermal abnormalities associated with aberrant apical E-cadherin localization explaining the epithelial fusion phenotypes observed in RIPK4 ${ }^{-1}$ mice and humans. In addition, we show that RIPK4 can modulate the actin cytoskeleton, for which an intimate link with cadherin regulation is known. ${ }^{14}$ These findings shed a new light on the underlying pathways causing different pterygium syndromes.

\section{Results}

RIPK4 deficiency leads to impaired keratinocyte differentiation and abnormal periderm retention. To elucidate the RIPK4 function in epidermal development, we analyzed the skin of RIPK4 ${ }^{-/-}$and RIPK4 ${ }^{+/+}$mouse littermates. We confirmed the reported $^{3}$ abnormal expression pattern of keratinocyte differentiation markers in E18.5 RIPK4 $^{-/-}$skin (Figure 1a). In contrast to wild-type (WT) skin, in which K14 expression is confined to the basal layer, we observed positive K14 staining in the outermost cell layers of RIPK4 ${ }^{-/-}$ skin, which shows their undifferentiated status. Strikingly, patchy areas of loricrin-positive corneocytes covered with a thick layer of undifferentiated cells were observed in RIPK4 ${ }^{-1-}$ skin (Figure 1a). Although differentiated corneocytes are observed in RIPK4 ${ }^{-1-}$ skin (Figures 1a and c), primary $\mathrm{RIPK}^{-1-}$ keratinocytes failed to differentiate in vitro in the presence of $\mathrm{Ca}^{2+}$ or vitamin $\mathrm{D}$, as shown by the lack of profilaggrin and caspase-14 expression (Figure 1b). Transmission electron microscopy analysis confirmed the occurrence of patches of cornified anuclear cells in RIPK4 ${ }^{-/}$skin (Figure 1c). In contrast to WT skin, the outermost epidermal cell layers are nucleated in RIPK4 ${ }^{-/}$skin (Figures 1a and c). This phenotype was originally described as parakeratosis, ${ }^{3}$ defined as keratinization with retention of nuclei. However, patches of anuclear corneocytes are found beneath the outermost nucleated cells (Figures 1a and c). Therefore, we reasoned that the outermost nucleated cells are not parakeratotic keratinocytes, but have a different origin. The outermost cell layers of RIPK4 ${ }^{-/}$skin have microvilli at the apical surface (Figure 1c, inset), a typical feature of periderm cells. ${ }^{1,2,15-17}$ The presence of both $\mathrm{K} 6$ and $\mathrm{K} 17^{15,16}$ confirmed the peridermal nature of the outermost skin layers in RIPK4 ${ }^{-1-}$ embryos (Figures $1 \mathrm{a}$ and $\mathrm{d}$ ), which persists in E18.5 RIPK4 $^{-1-}$ mice, whereas isolated E18.5 WT littermate embryos are devoid of periderm (Figure 1a). In contrast to $\mathrm{RIPK}^{+/+}$littermates, abnormal suprabasal and peridermal proliferation is observed in E16.5 $\mathrm{RIPK}^{-1-}$ skin, as shown by the presence of BrdU positivity, multiple p63-positive nuclei and peridermal marker K17 staining ${ }^{15}$ (Figure 1d, lower panels). At E17.5, epidermal proliferation was restricted to the basal layer both in RIPK4 ${ }^{+/+}$and RIPK4 ${ }^{-/-}$epidermis (Supplementary Figure S1). These observations suggest that differentiation-induced proliferation arrest of suprabasal cells is delayed in $\mathrm{RIPK}^{-/-}$epidermis.
RIPK4 is required for skin barrier formation. An abnormal skin architecture resembling that of RIPK $4^{-/}$mice was also reported for $\mathrm{IRF}^{-1-}$ mice, which have a defective skin barrier. ${ }^{10,11}$ In WT mice, the skin barrier starts forming at E16 and is fully functional at E17. ${ }^{18}$ Dye exclusion assays showed that toluidine blue and lucifer yellow penetrate E18.5 $\mathrm{RIPK}^{-/-}$skin but not RIPK4 ${ }^{+/+}$skin (Figures $2 \mathrm{a}$ and b), indicating that a mature outside-in barrier is not formed in RIPK4 $^{-/-}$skin. In E18.5 RIPK4 ${ }^{+/+}$embryos, the dermally injected biotin tracer was stopped at the transition of the upper granular layer to the cornified layer (Figure 2c), whereas in RIPK4 ${ }^{-1-}$ skin the biotin tracer diffused to the top layers of the skin, showing that a functional inside-out barrier was absent. Interestingly, the biotin tracer was excluded from the cornified patches in $\mathrm{RIPK}^{-1-}$ skin, suggesting the presence of a functional barrier at these patches (Figure 2c). Taken together, these results demonstrate unequivocally that RIPK4 is important for normal skin barrier formation.

\section{RIPK4 $^{-I-}$ mice have multiple abnormal epithelial fusions} characterized by aberrant apical E-cadherin localization. The most striking characteristic of RIPK $4^{-/-}$mice is the epithelial fusion of all external orifices and the esophagus, as well as the partial attachment of limbs to the body wall ${ }^{3}$ (Figures 3a-f), but it remains unclear how these fusions arise. When we analyzed different embryonic stages, we found that the mouth cavity is initially formed in E12.5 RIPK4 ${ }^{-/-}$ embryos, but the epithelia lining the oral cavity fuse completely at E13.5 (Supplementary Figures S2F and G). Skeletal staining and histological sections showed that $\mathrm{RIPK}^{-1-}$ mice have cleft palate (Figure $3 \mathrm{~b}$ and Supplementary Figure S2J). We believe that this is caused by fusion of the tongue to the developing palatal shelves, which prevents the formation of the secondary palate (Supplementary Figures S2B-D, G, H and I).

We hypothesized that the fusions could be caused by abnormal periderm adhesion. We found that the adhesion molecule E-cadherin, which is absent from the apical side of $\mathrm{RIPK}^{+/+}$peridermal cells, was frequently localized apically in K17-positive RIPK4 ${ }^{-1-}$ peridermal cells lining the oral cavity, esophagus and skin of RIPK4 ${ }^{-1-}$ mice (Figures $3 i, k, m$ and $n$ ). Ectopic localization of E-cadherin at the apical surface of two apposed epithelia could cause them to adhere and subsequently fuse. This likely explains the intraoral and esophageal fusions and the partial attachment of the limbs to the body wall in $\mathrm{RIPK}^{-/-}$mice (Figures $3 b, d$ and $\mathrm{f}$ ). Similar to the hypoplastic external genitalia observed in humans affected by PPS, ${ }^{8,9}$ the genital tubercle was lacking in $\mathrm{RIPK}^{-1-}$ mice (Figures $3 f$ and $4 a)$.

Morphogenetic defects upon RIPK4 depletion in Xenopus embryos. To further investigate the developmental function of RIPK4, we used the Xenopus tropicalis frog model. We depleted zygotic $X$. tropicalis RIPK4 (xRIPK4) by using a splicing-blocking morpholino (xRIPK4Mo $\left.\mathrm{E}_{\mathrm{E} 12}\right)$. The efficacy of this morpholino was shown by RT-PCR analysis, which revealed a concentration-dependent reduction of the WT xRIPK4 transcript (630 bp) together with the appearance of fragments representing aberrant splicing products 
a

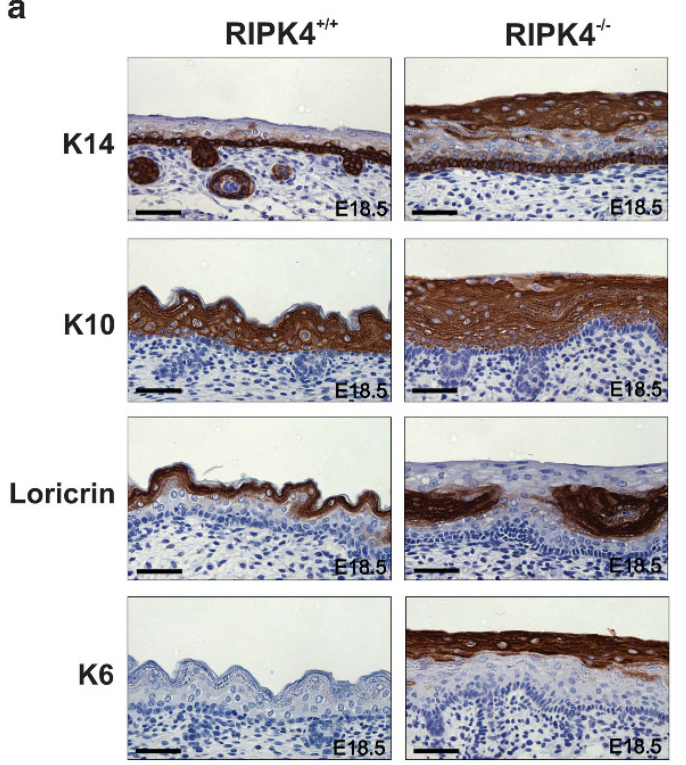

C

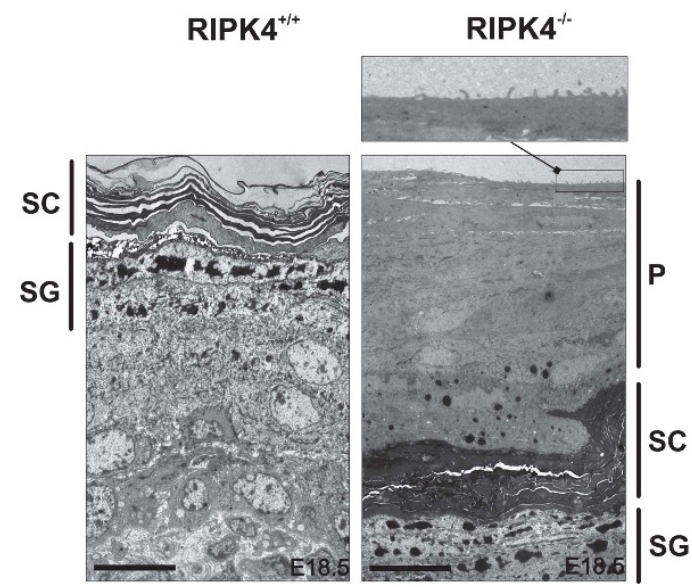

b

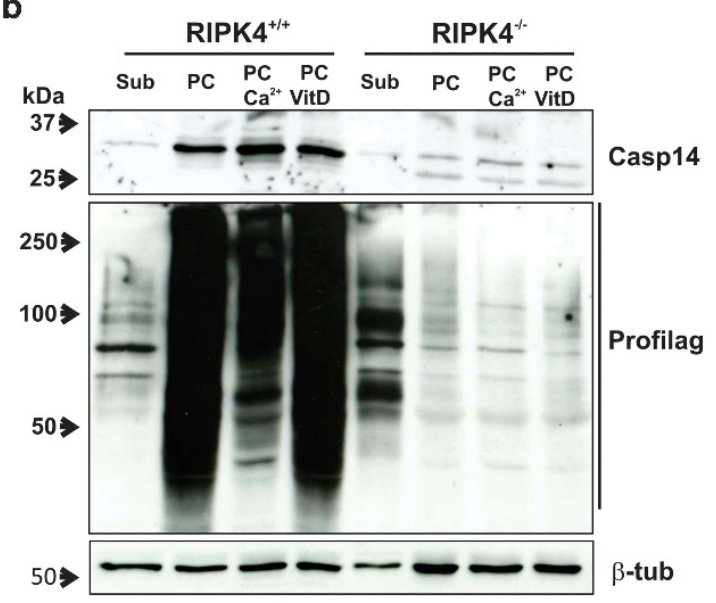

d

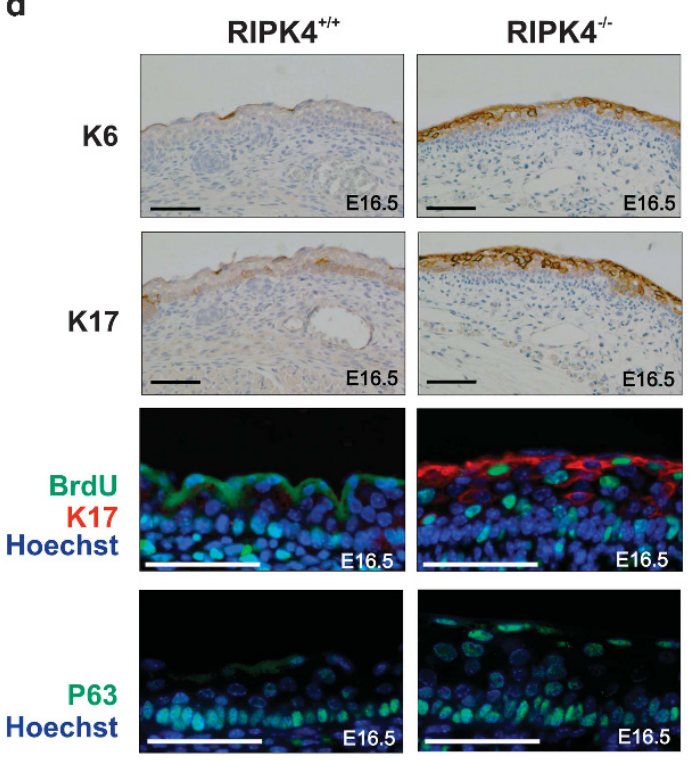

Figure 1 Skin phenotype of RIPK4 ${ }^{-1-}$ mice. (a) Immunohistochemical analysis of keratinocyte differentiation markers in E18.5 skin. Keratin 14 (K14), K10 and loricrin are markers of undifferentiated keratinocytes, intermediate keratinocyte differentiation and terminal keratinocyte differentiation, respectively. Note the presence of a thick superficial layer of immature, K6-positive nucleated cells in RIPK4 ${ }^{-1-}$ skin that is absent in RIPK4 ${ }^{+/+}$littermates and the interspersed patches of differentiated keratinocytes that are found internally in RIP4 ${ }^{-1-}$ skin. (b) In vitro differentiation of primary RIPK4 ${ }^{+/+}$and RIPK4 ${ }^{-1-}$ keratinocytes. Primary keratinocytes were isolated from E18.5 embryos and induced to differentiate by postconfluent growth $(\mathrm{PC})$ or $\mathrm{PC}$ growth combined with the addition of calcium $\left(\mathrm{Ca}^{2+}\right)$ or vitamin $\mathrm{D}(\mathrm{VitD})$. Undifferentiated subconfluent cultures $(\mathrm{Sub})$ served as control. Keratinocyte cultures of RIPK4 ${ }^{-1-}$ and RIPK4 ${ }^{+/+}$littermates were compared by immunoblotting for filaggrin (filag) and caspase-14 (casp14) as differentiation markers. (c) Electron micrographs of E18.5 RIPK4 ${ }^{+/+}$and RIPK4 ${ }^{-l-}$ skin. Note the presence of microvilli on superficial periderm cells of RIPK4 ${ }^{-1-}$ skin (inset). (d) Immunostaining for periderm markers: K6 and K17, cell proliferation analysis by BrdU staining (green) and immunofluorescence analysis of p63 (green) at E16.5. Note abnormal suprabasal and peridermal BrdU- and p63-positive nuclei in RIPK4 ${ }^{-I}$ skin. For cell proliferation analysis at different embryonic stages, see Supplementary Figure S1. Scale bars: (a and d) $50 \mu \mathrm{m}$ and (b) $10 \mu \mathrm{m}$. P, periderm; SC, stratum corneum; SG, stratum granulosum

(Supplementary Figure S3A). The embryos depleted with XRIPK4Mo $\mathrm{E} 212_{2}$ developed normally through gastrulation and reached the tadpole stages (Figure $4 \mathrm{a}$, middle panels), but consistently displayed swelling of the cloacal tube after Nieuwkoop stage 40 (Figure 4a, lower right panel) because of the inability to clear the pronephric filtrate, indicating a closed cloaca opening. This phenotype resembles the anal fusion observed in RIPK4 ${ }^{-1-}$ mice. All embryos injected with control morpholino had formed a functional cloacal tube (Figure 4a, upper right panel).

In addition to the splicing-blocking morpholino, we also used a translation-blocking morpholino $\left(x R I P K 4 \mathrm{Mo}_{\text {atg }}\right)$ that blocks translation of zygotic and maternal transcripts. Surprisingly, injection of $x R I P K 4 M_{a}$ atg induced a severe gastrulation defect (Figures 4b-d). The RIPK4-depleted embryos had a wide open blastopore and displayed complete exogastrulation, consequently failing to internalize the endodermal and mesodermal tissues and lacking axial features (Figure $4 b$, second panel). This gastrulation block was completely rescued in $70 \%$ of the embryos by coinjection of hRIPK4 mRNA but not by coinjection of mRNA coding for catalytically inactive hRIPK4K51R mutant $^{19-21}$ (Figures $4 \mathrm{~b}$ (third and fourth panel) and $\mathrm{C}$ and Supplementary Figure S3C), showing that the phenotype is specific and depending on the RIPK4 kinase activity. 
a

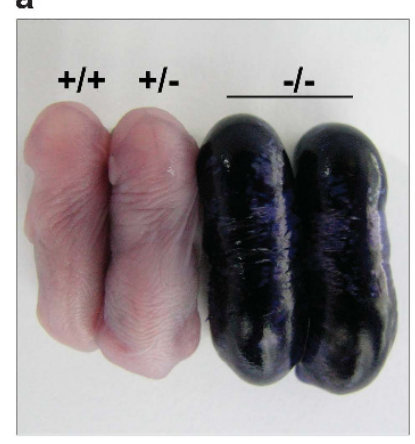

b

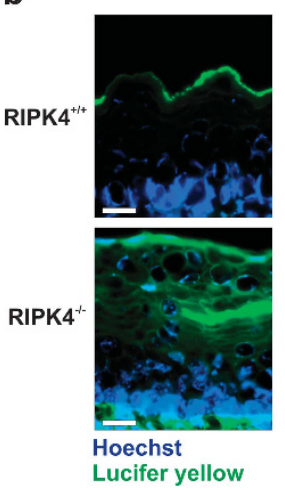

C

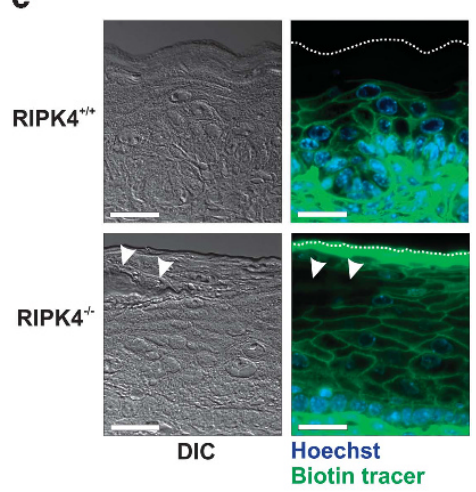

Figure 2 Defective skin barrier in E18.5 RIPK4 ${ }^{-1-}$ mice. (a) Toluidine blue and (b) lucifer yellow dye exclusion assays to evaluate skin barrier in E18.5 RIPK4 ${ }^{-1-}$ and $\mathrm{RIPK}^{+/+}$littermates. Both dyes penetrate RIPK4 ${ }^{-1-}$ skin but not RIPK4 ${ }^{++}$skin. (c) Inside-out skin barrier was analyzed by dermal injection of a biotin tracer followed by streptavidin staining (green). Nuclei were stained with Hoechst (Blue). The biotin tracer is stopped at the transition of the granular layer to the cornified layer in RIPK4 ${ }^{+/+}$skin, but migrates until the outer skin surface in RIPK4 ${ }^{-l-}$ skin. The biotin tracer is excluded from cornified patches (arrowheads) in RIPK4 ${ }^{-1-}$ skin. Scale bars: $20 \mu \mathrm{m}$

To rule out that the severe gastrulation defect is secondary to a cell fate change, we analyzed expression of the dorsal organizer gene chordin by whole-mount in situ hybridization. The expression pattern of this marker was normal in xRIPK4Moatg-injected embryos, although the domain of chordin expression was consistently narrower in RIPK4 morphants, indicative of mild ventralization (Supplementary Figure S3B). This is in line with the reported expanded domain of chordin expression observed upon overexpression of RIPK4 in Xenopus that could be due to increased $\mathrm{Wnt} / \beta$ catenin signaling. ${ }^{22}$ However, it is important to note that embryos that are completely ventralized by depletion of maternal $\beta$-catenin do still gastrulate. ${ }^{23}$ Hence, we believe that the strong gastrulation phenotype we observe cannot be attributed to aberrant Wnt signaling alone or cell fate changes in general and is the result of aberrant morphogenetic movements.

Epiboly during amphibian gastrulation involves the thinning and spreading of the multilayered animal cap cells. The rapid expansion of ectodermal surface to cover the embryo and engulf the endoderm that is required during epiboly is achieved by the radial intercalation of several cell layers in the blastocoel roof (BCR), causing its thinning. ${ }^{24-27}$ In contrast to control-injected embryos, we noticed that the BCR of xRIPK4Mo ${ }_{\text {atg }}$-depleted embryos showed impaired thinning of the ectodermal cell layers (Figure $4 d$ ), suggesting that this is the onset of a disrupted epibolic movement hampering gastrulation.

RIPK4 overexpression affects cellular morphology in a kinase-dependent manner. To study the effect of hRIPK4 overexpression in keratinocytes, we transduced doxycycline (dox)-inducible hRIPK4-Flag constructs in the HaCaT keratinocyte cell line. Inducible overexpression of luciferase-Flag was used as a negative control. Although parental and control-transfected HaCaT cells grew in characteristic round islands with cells showing stable contacts and minimizing their cell-free borders, hRIPK4WT-expressing cells acquired a more scattered and fusiform morphology (Figure 5a). Phalloidin staining in control-transfected cells showed tight cell-cell contacts and filamentous-actin (F-actin) concentrated in parallel bundles at the cell-free borders of the islands. In contrast, the loosely arranged cells overexpressing WT RIPK4 had scarce cell-cell contacts and F-actin primarily organized in stress fibers and in filopodial and lamellipodial extensions (Figure 5b). Although this phenotype resembles epithelial-mesenchymal transition, we did not observe changes in the expression levels of E-cadherin or mesenchymal markers such as vimentin (Supplementary Figure S4). The change in cellular morphology was dependent on the RIPK4 kinase activity (Figures $5 \mathrm{a}$ and b). To further address the role of RIPK4 in actin cytoskeleton organization, we stained $\mathrm{RIPK}^{+/+}$and $\mathrm{RIPK}^{-/-}$skin sections for F-actin. RIPK4 ${ }^{+/+}$epidermis displayed a membrane-associated F-actin network that was enriched at cell-cell contacts, but this cortical network was strongly reduced or absent in RIPK $4^{-1-}$ epidermal cells (Figure 5c). These data confirm the importance of RIPK4 for correct cytoskeletal organization.

\section{Effects of RIPK4 mutants on cell scattering and Xenopus} gastrulation. To address the functional properties of RIPK4 mutants identified in BPS, ${ }^{8,9}$ we cloned four of these mutants: one introducing a frameshift and premature stop codon in the kinase domain (hRIPK4ins777a), one introducing a stop at the end of the intermediate domain (hRIPK4S376X), and two missense mutants in the kinase domain (hRIPK4I121N and hRIPK4T184I) (Figure 5f). HaCaT cells were transduced with these Flag-tagged, dox-inducible hRIPK4 constructs together with an NF-kB reporter. Overexpression of hRIPK4WT led to cell scattering associated with the appearance of multiple lamellipodia and cytoskeletal rearrangements (Figures $5 \mathrm{a}$ and d). but the BPS-associated hRIPK4I121N; T184I and ins777a mutants did not induce changes in cell morphology or actin organization (Figure $5 d$ and Supplementary Figure S5). Overexpression of a truncation mutant with an intact kinase domain (hRIPK4S376X) did cause cell scattering (Figure 5d and Supplementary Figure S5). Interestingly, the latter mutant could still activate NF-kB, while mutants with a mutant kinase domain failed to do so (Figure 5e).

To study the functional properties of the BPS-associated RIPK4 mutants in vivo, we tried to complement the gastrulation defect in xRIPK4Moatg-injected $X$. tropicalis embryos by 

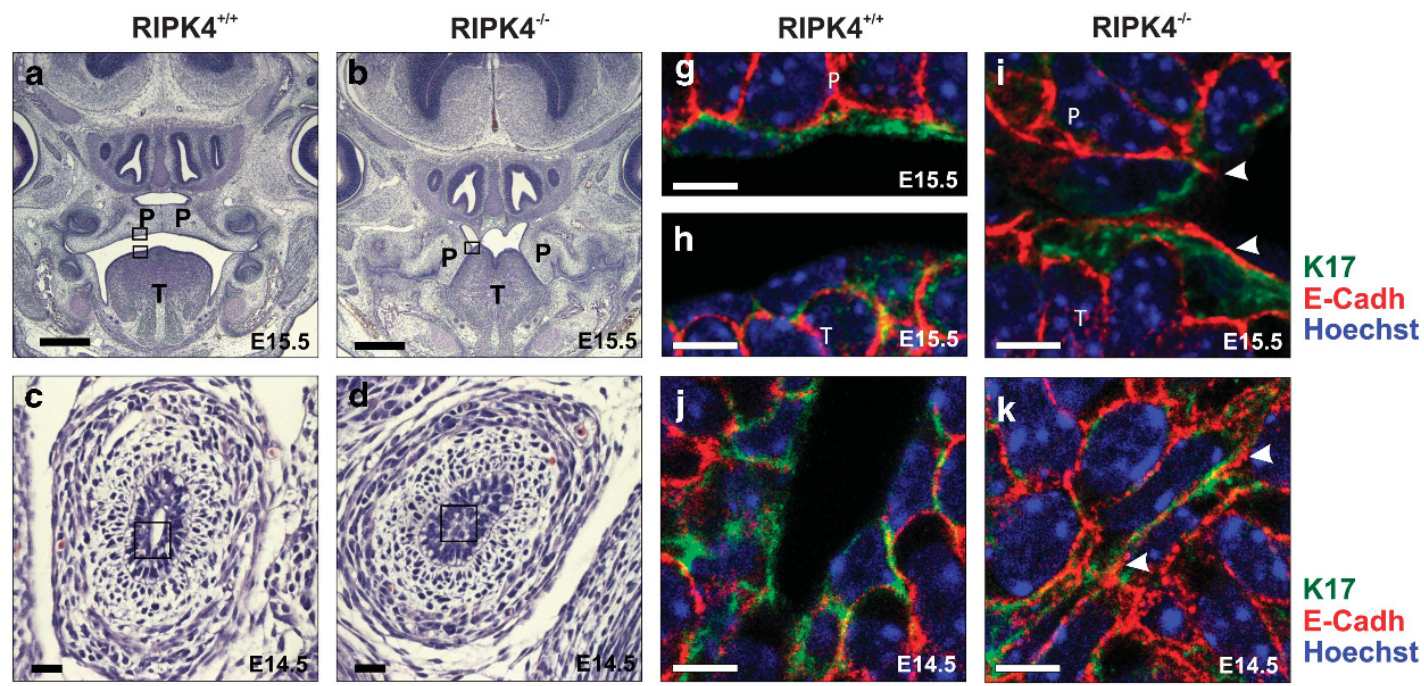

K17

E-Cadh

Hoechst
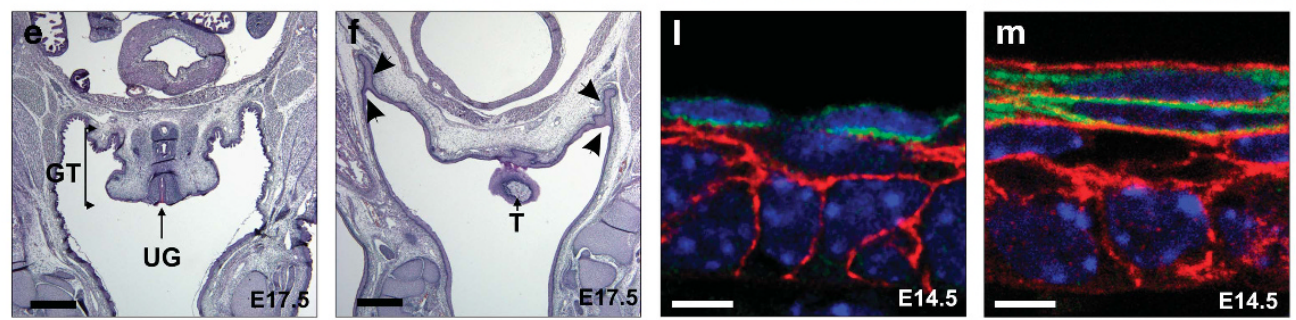

K17

E-Cadh

Hoechst
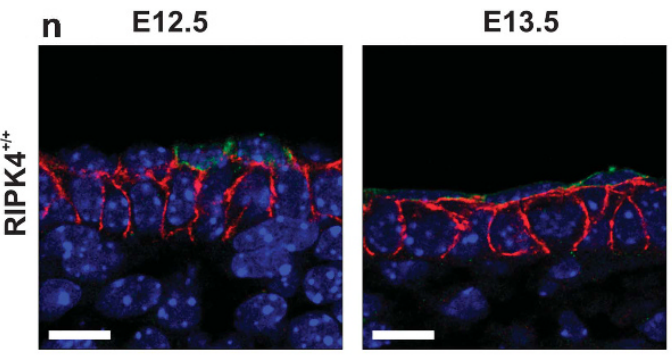

E14.5
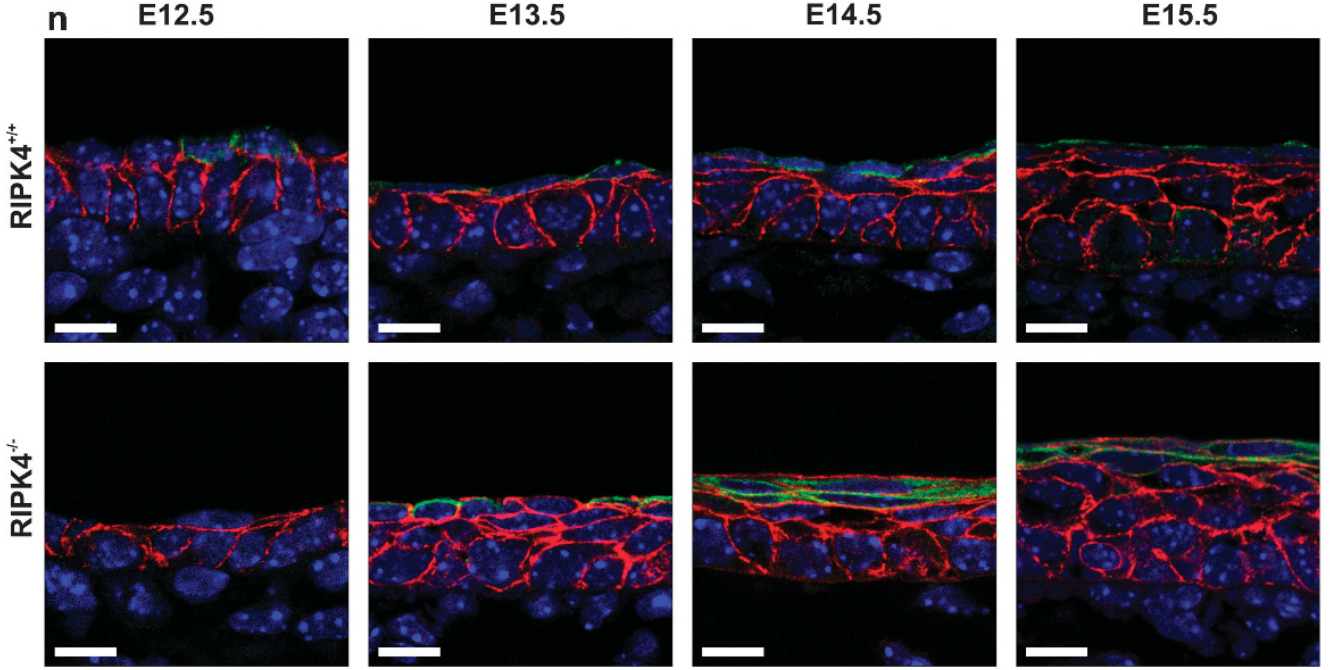

K17

E-Cadh

Hoechst

Figure 3 Epithelial fusions and ectopic E-cadherin expression in RIPK4 ${ }^{-1-}$ mice. (a-f) Histological analysis of sections from E15.5 head (a and b), E14.5 esophagus (c and d) and E17.5 hind limbs (e and $\mathbf{f})$ of RIPK4 ${ }^{+/+}(\mathbf{a}, \mathbf{c}$ and $\mathbf{e})$ and RIPK4 ${ }^{-1-}(\mathbf{b}, \mathbf{d}$ and $\mathbf{f})$ littermates. (a) Elevated and fused palatal shelves $(\mathrm{P})$ in RIPK4 ${ }^{+/+}$mice. (b) Failure to elevate

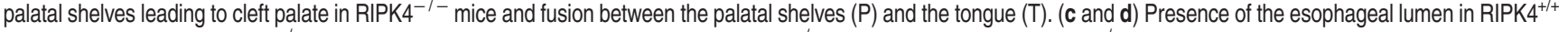

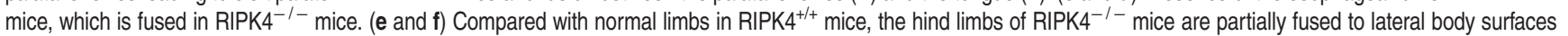
(arrowheads). Similar observations were carried out for the forelimbs (data not shown). Note the absence of the genital tubercle (GT) and urogenital groove (UG) in RIPK4 ${ }^{-1-}$ mice. T, tail. (g-m) Immunofluorescence analysis of the fusion phenotypes in RIPK4 ${ }^{-I}$ mice compared with RIPK4 ${ }^{+/+}$littermates: E-cadherin (E-cadh) (red), keratin 17 (K17) (green) and nuclei by Hoechst (blue). In RIPK4 ${ }^{+/}$embryos, E-cadh is absent from the apical side of superficial periderm cells $(\mathbf{g}, \mathbf{h}, \mathbf{j}$ and $\mathbf{I})$, but present at the apical side in

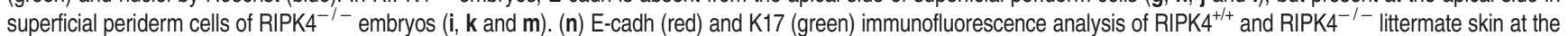
indicated developmental stages. Scale bars: (a, b, e and f) $500 \mu \mathrm{m}$; (c and d) $20 \mu \mathrm{m} ;(\mathbf{g}-\mathbf{m}) 5 \mu \mathrm{m}$; and (n) $10 \mu \mathrm{m}$

coinjecting in vitro transcribed mRNA coding for several of the disease-related human RIPK4 (hRIPK4) mutants. As expected, WT hRIPK4 rescued the gastrulation block. In contrast, none of the clinical RIPK4 mutants was able to do so (Figure 6 and Supplementary Figure S6). The hRIPK4ins777a mutant showed a lower expression level, suggesting that the mRNA or protein of this mutant is less stable.

In conclusion, our experiments indicate that RIPK4-induced cell scattering is related to rearrangement of the actin cytoskeleton. Moreover, both in keratinocyte cell cultures 

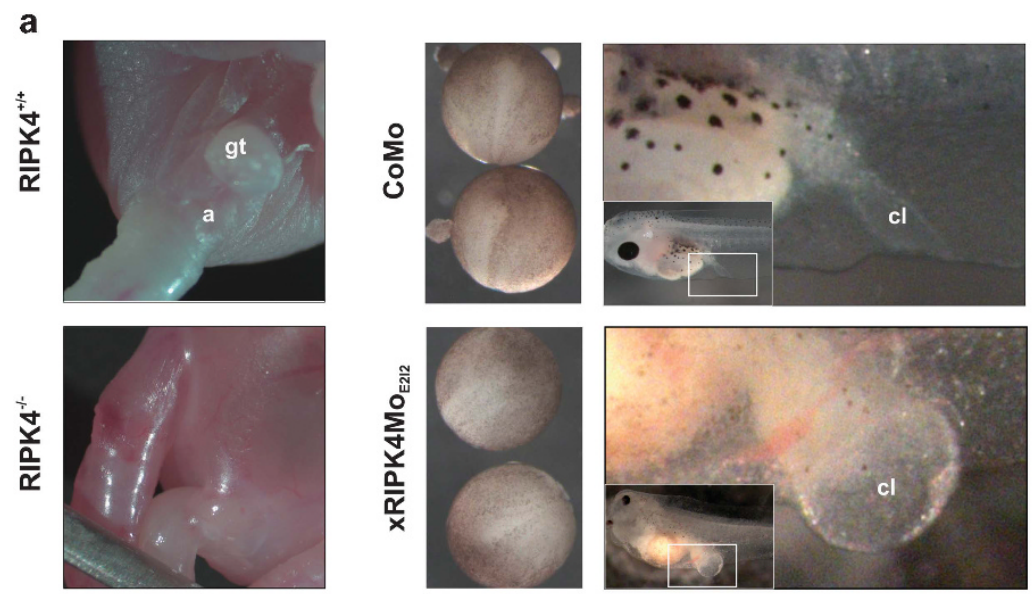

b
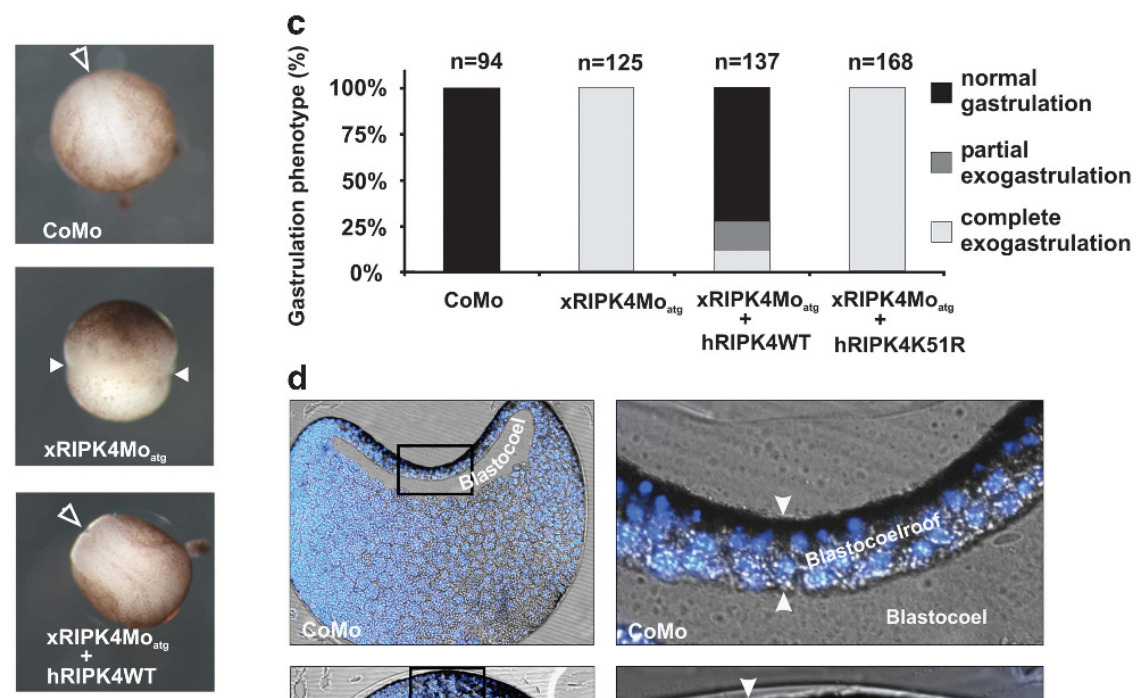

d hRIPK4WT hRIPK4K51R
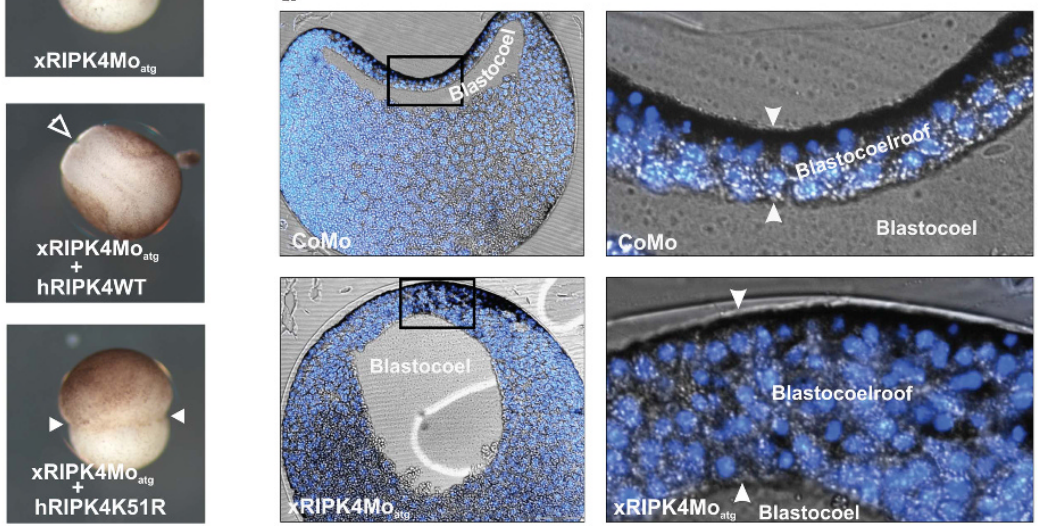

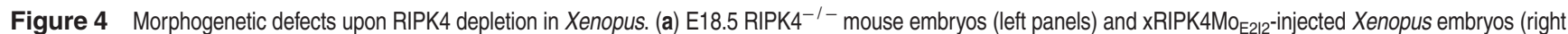
panels) showing anal atresia and cloacal fusion, respectively. The genital tubercle (gt) and anal opening (a) are absent in RIPK4 ${ }^{-1-}$ mice (a, lower left panel). X. tropicalis embryos injected at the two-cell stage with $20 \mathrm{ng}$ of $x \mathrm{XIPK}_{\mathrm{M}} \mathrm{MO}_{\mathrm{E} 212}$ or $20 \mathrm{ng}$ of control morpholino (CoMo) develop normally through gastrulation (middle panels, embryos shown

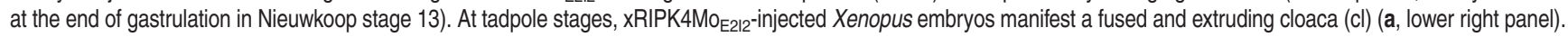
Efficiency of the XRIPK4MO ${ }_{E 212}$ morpholino was shown by RT-PCR, see Supplementary Figure S3A. (b and c) Gastrulation defect upon depletion of xRIPK4 with a translationblocking morpholino (xRIPK4Mo ${ }_{\text {atg }}$ ) and rescue experiments by hRIPK4WT and an hRIPK4K51R mutant. Embryos were injected at the two-cell stage with $10 \mathrm{ng}$ of CoMo or xRIPK4Mo $\mathrm{O}_{\mathrm{atg}}$, alone or in combination with $250 \mathrm{pg}$ RNA coding for myc-tagged hRIPK4WT or hRIPK4K51R. Although CoMo-injected embryos have completely closed their blastopore at the Nieuwkoop stage 13 (open arrowhead in b), $100 \%$ of sibling embryos depleted for RIPK4 (XRIPK4MOATG) manifest a completely open blastopore, only showing circumferential constriction in the equator (filled arrowheads in b). Coinjection of hRIPK4WT completely rescues the gastrulation block, as witnessed by the closed blastopore (b, third panel open arrowhead) in $70 \%$ of the embryos, while expression of a kinase-dead point mutant hRIPK4K51R does not (b, fourth panel). (c) Graph showing percentages of gastrulation phenotypes for corresponding injections in Xenopus. Data represent pooled data of two independent experiments, and total number of injected embryos is indicated above each bar as ' $n$ '. (d) Defective BCR thinning in xRIPK4Moatg-injected Xenopus embryos. Sections are shown with the animal pole of the embryo oriented to the top. White arrowheads indicate thickness of the BCR (left panels). Nuclei were stained with Hoechst (blue)

and in Xenopus, the BPS-associated hRIPK4 mutants behave like the experimental kinase-dead mutant, except for the S376X mutant in keratinocytes.

Ectopic RIPK4 expression rescues IRF6 deficiency. The $\mathrm{RIPK}^{-/-}$mouse phenotype we observed is similar to that of the $\mathrm{IRF}^{-1-}$ and the IRF6 ${ }^{\mathrm{R} 84 \mathrm{C} / \mathrm{R} 84 \mathrm{C}}$ mice. ${ }^{10,11}$ Moreover, similar to RIPK4 activity, IRF6 is also required for gastrulation in Danio rerio and $X$. laevis. ${ }^{28}$ Until now, there is no evidence that functionally connects these two molecules. A hRIPK4 promoter reporter construct was significantly induced by cotransfection of hIRF6 in mammalian cells, showing that RIPK4 can act downstream of IRF6 (Figure 7a). Furthermore, $X$. tropicalis embryos injected with a dominant-negative IRF6 
a
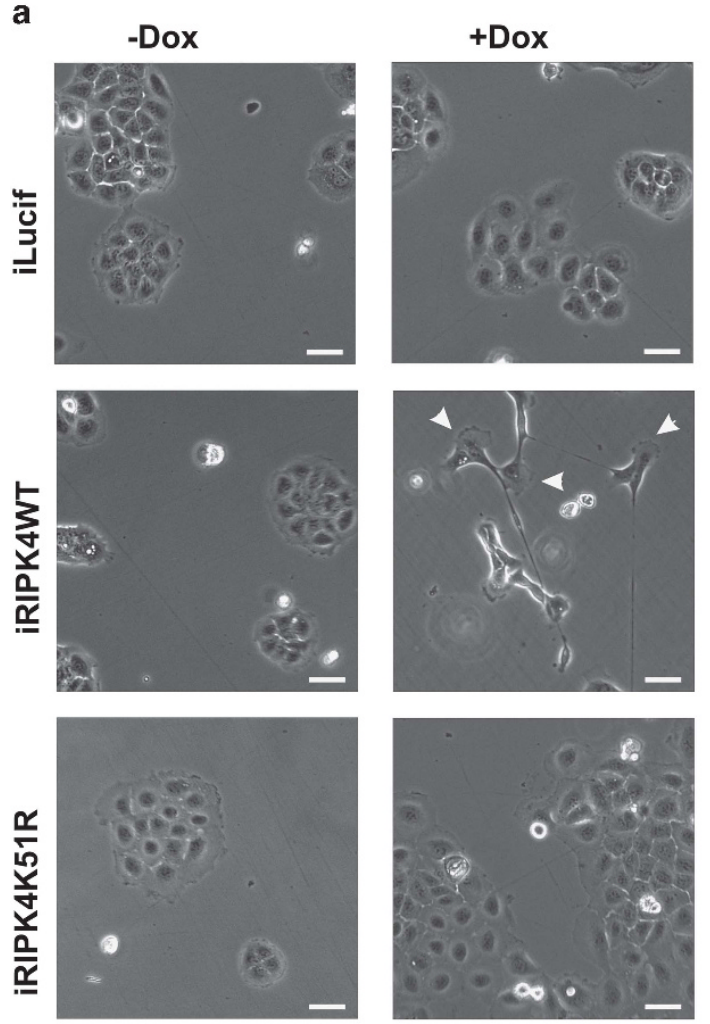

c

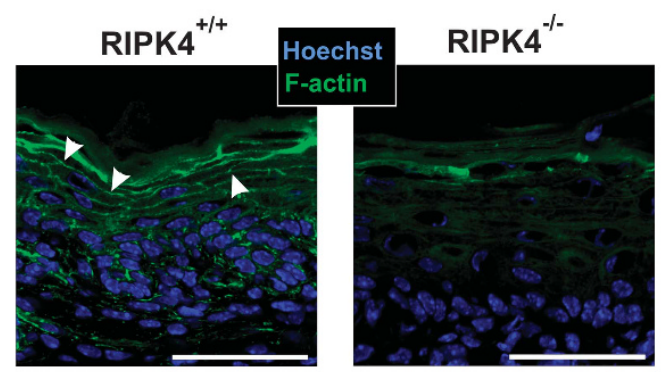

e

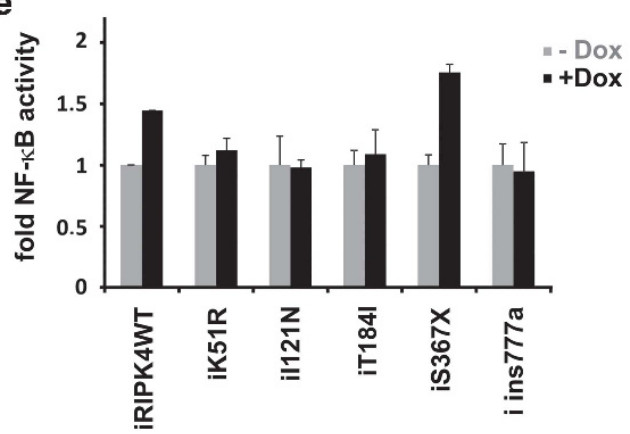

b
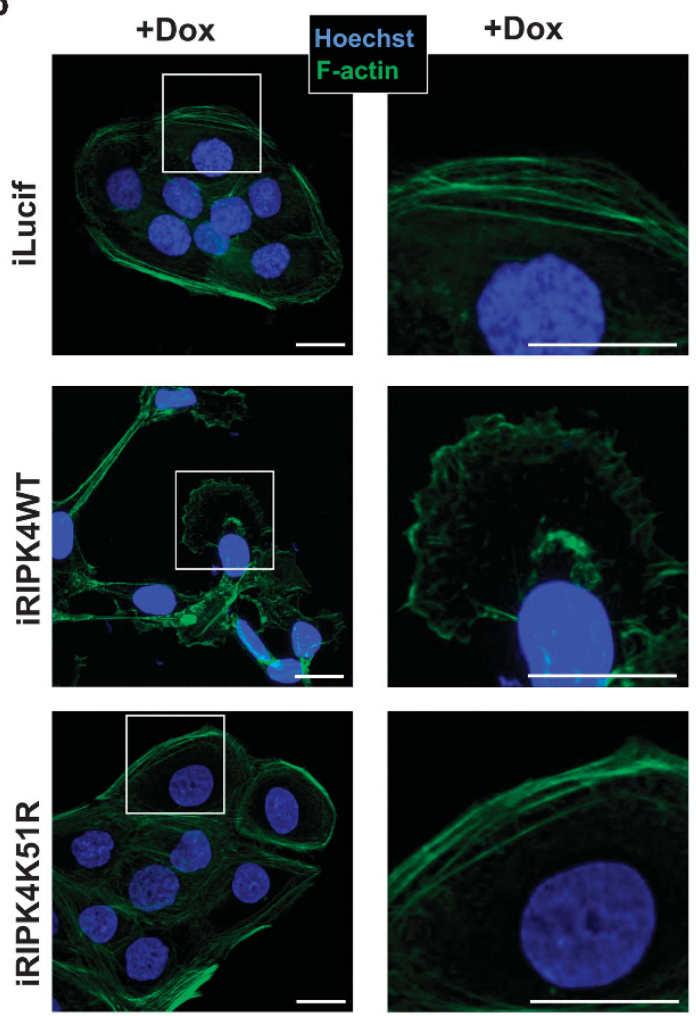

d
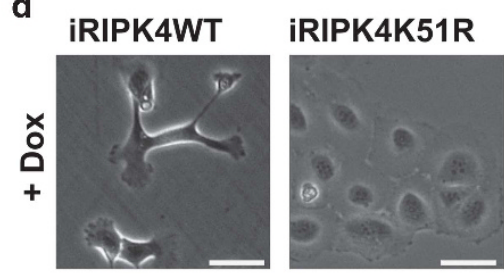

iRIPK4I121N
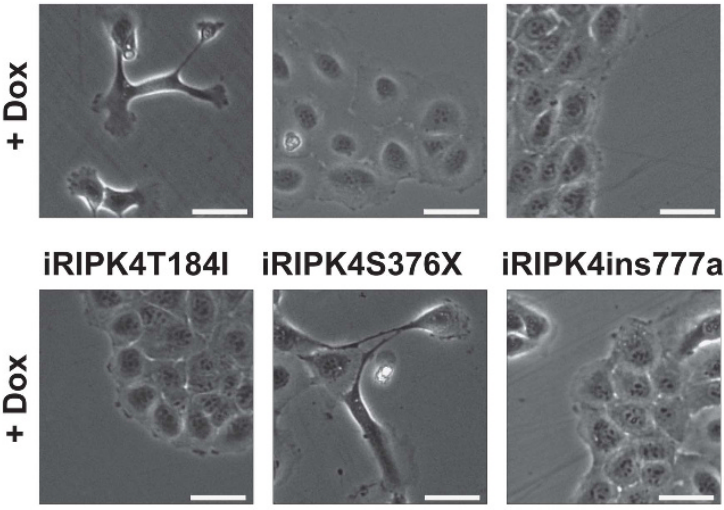

f

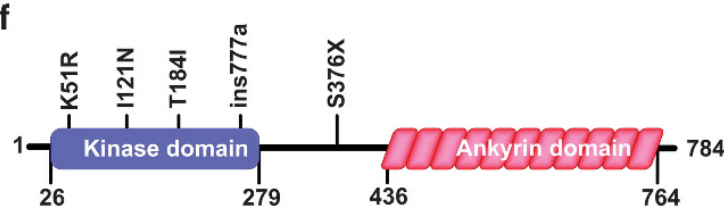

Figure 5 Induction of cell scattering and cytoskeletal rearrangements by kinase-active RIPK4. (a) HaCaT cells transduced with dox-inducible expression vectors for luciferase, hRIPK4WT or hRIPK4K51R were treated with $1 \mu \mathrm{g} / \mathrm{ml}$ dox for $48 \mathrm{~h}$ and cell scattering was analyzed by phase contrast microscopy. Note the elongated shape and the formation of extensive lamellipodia in the scattering cells (arrowheads). (b) Phalloidin staining of the F-actin network in HaCaT cells overexpressing the indicated transgenes. Boxed areas in the left panels are enlarged in the corresponding right panels. (c) Frozen sections of $18.5 \mathrm{dpc} \mathrm{RIPK4^{+/+ }}$ and RIPK4 ${ }^{-/-}$skin were stained with Phalloidin-TRITC. Phalloidin-TRITC staining was pseudocolored in green. Note the presence of the membrane-associated F-actin network in RIPK4 ${ }^{+/+}$epidermal cells (arrowheads) that is largely absent in RIPK4 ${ }^{-1-}$ epidermal cells. (d) HaCaT cells were transduced with dox-inducible hRIPK4WTor with the indicated hRIPK4 mutant constructs. Cell scattering, analyzed by phase contrast microscopy $48 \mathrm{~h}$ after induction with $1 \mu \mathrm{g} / \mathrm{ml}$ dox (see also Supplementary Figure S5B). (e) NF- $\kappa$ B activity in doxycline-induced hRIPK4WT- or hRIPK4 mutantsexpressing $\mathrm{HaCaT}$ cells. Data are represented as mean relative luciferase activity normalized to non-induced $+/-s . d$. from a representative experiment performed in triplicate. Protein levels of the indicated constructs are shown in Supplementary Figure S5B. (f) Schematic overview of the different RIPK4 mutations tested. Scale bars: $50 \mu \mathrm{m}$ 
a

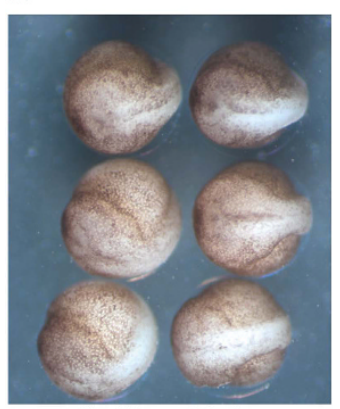

CoMo + $\beta$ Gal

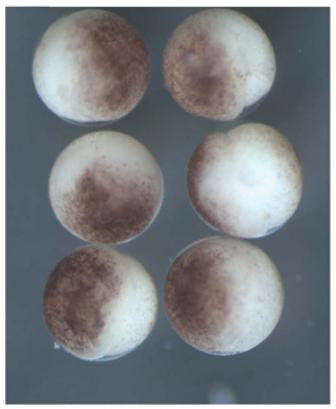

$x R I P K 4 M o_{\text {atg }}+h R I P K 4 I 121 \mathrm{~N}$

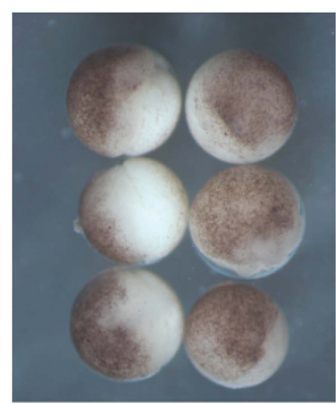

$x R I P K 4 \mathrm{Mo}_{\text {atg }}+\beta \mathrm{Gal}$

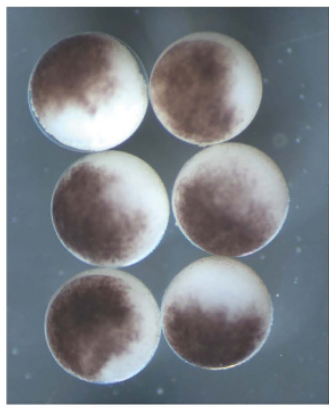

xRIPK4Mo atg $_{\text {a }}+$ hRIPK4T184I

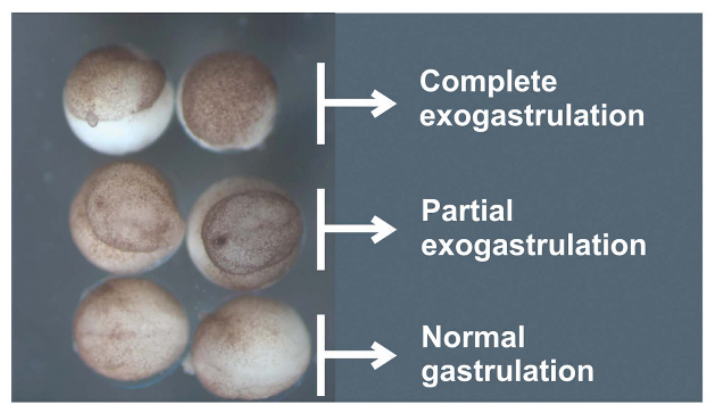

xRIPK4Mo $\mathrm{o}_{\text {atg }}+\mathrm{hRIPK4WT}$
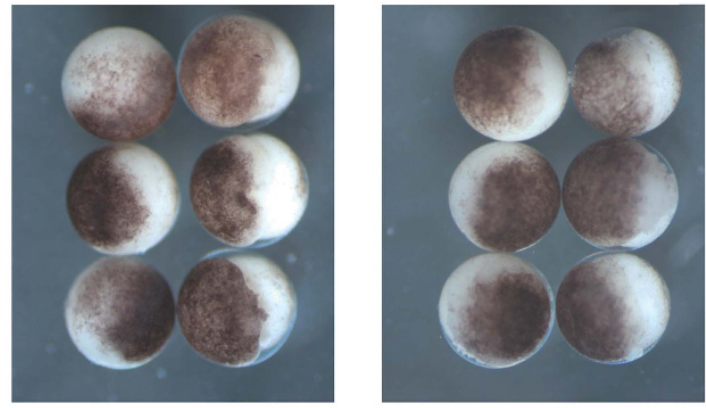

xRIPK4Mo atg $_{\text {atg }}+$ hRIPK4S376X xRIPK4Mo atg $_{\text {atg }}$ hRIPK4i777a

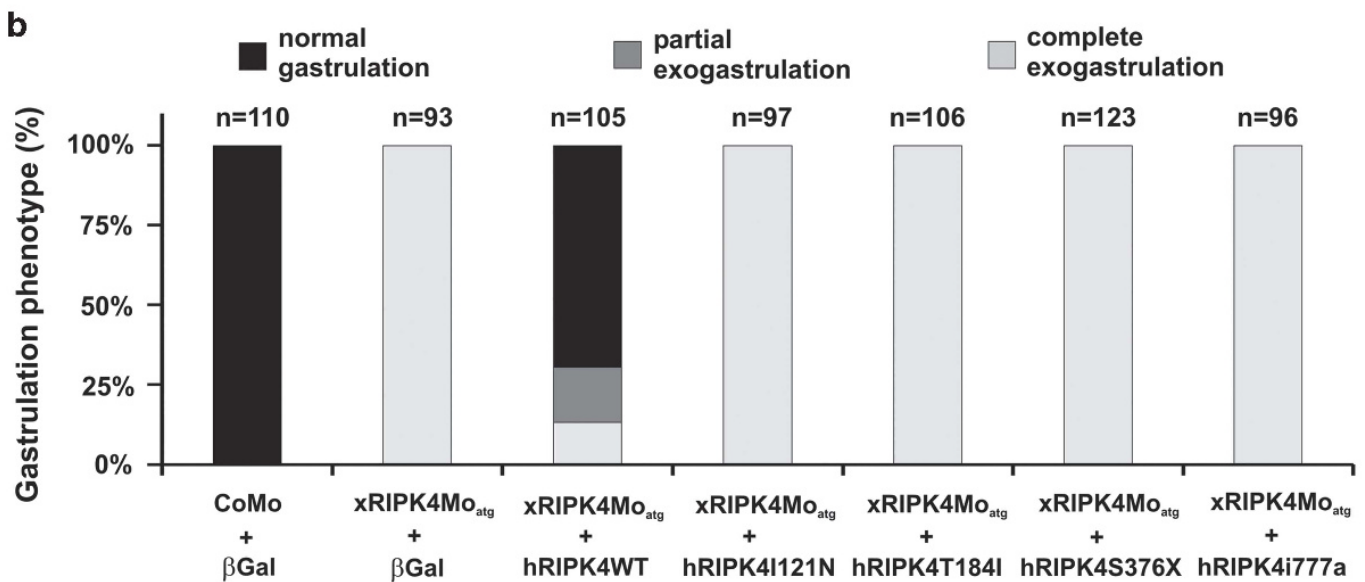

Figure 6 Effects of RIPK4 mutants on cell scattering and Xenopus gastrulation. (a and $\mathbf{b}$ ) Bartsocas-Papas-associated hRIPK4 mutants fail to rescue the gastrulation phenotype mediated by XRIPK4Mo atg depletion in Xenopus embryos. Phenotypic analysis of the gastrulation defect of embryos injected with $10 \mathrm{ng}$ of CoMo or XRIPK4 Mo atg combined with $250 \mathrm{pg}$ of the indicated mRNA transcripts. (b) Quantification of the experiment as shown in (a) summarizing the percentages of injected embryos with corresponding phenotypes. The total number of injected embryos is indicated above each bar as ' $n$.' Expression of the coinjected hRIPK4 transcripts was shown by western blot (Supplementary Figure S6)

construct (IRF6DBD (IRF6 DNA-binding domain) $)^{28}$ showed reduced RIPK4 mRNA expression (Figure 7b), further confirming that RIPK4 is transcriptionally regulated by IRF6. Injection of in vitro transcribed IRF6DBDexpressing mRNA caused severe exogastrulation in $X$. tropicalis embryos (Figures 7c (middle panel) and d), as reported previously for $X$. laevis and $D$. rerio. ${ }^{29}$ Interestingly, coinjection of hRIPK4 mRNA completely rescued this gastrulation defect in $20 \%$ of the embryos (Figures 7c (lower panel) and d) and ameliorated gastrulation in the remaining $80 \%$. This indicates that the disruption of gastrulation by IRF6DBD is at least partially due to suppression of RIPK4. Interestingly, it was reported that disruption of gastrulation movements by the expression of IRF6DBD in zebrafish embryos was accompanied by a substantial reduction of cortical actin in the superficial cell layer. ${ }^{28}$ Therefore, we performed actin staining on stage 11 animal caps isolated from embryos injected with either RIPK4Mo $\mathrm{Mtg}_{\text {atg }}$ or IRF6DBD RNA. Although control morpholinoinjected animal caps showed high levels of cortical actin in the cells facing the blastocoel, little F-actin was present in similar stage animal caps injected with RIPK4Mo atg or IRF6DBD RNA (Figure 7e). This further confirms that the phenotypes of embryos injected with either RIPK4Mo atg or IRF6DBD RNA are due to comparable cellular dysfunctional processes. 
a

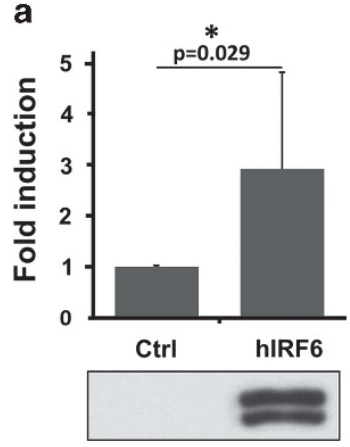

b

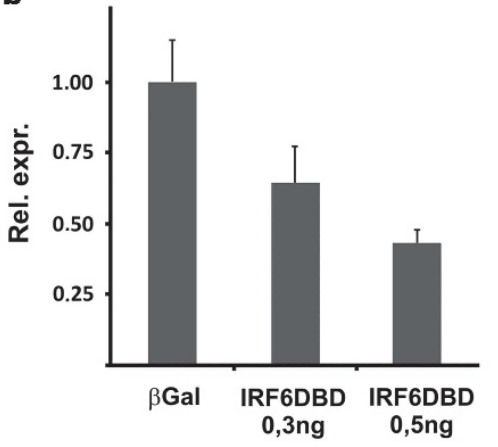

d

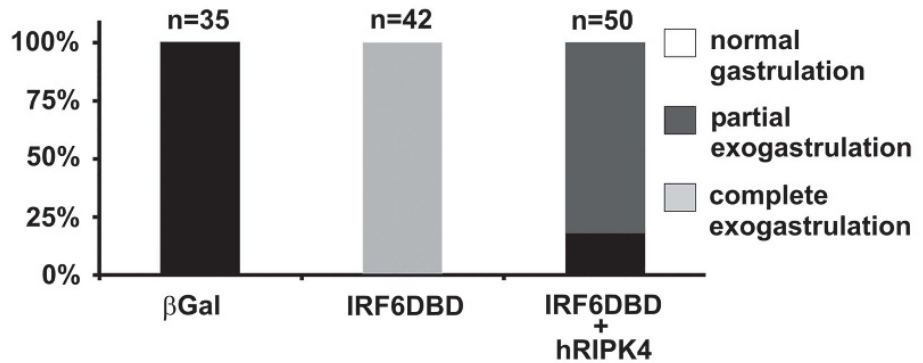

e

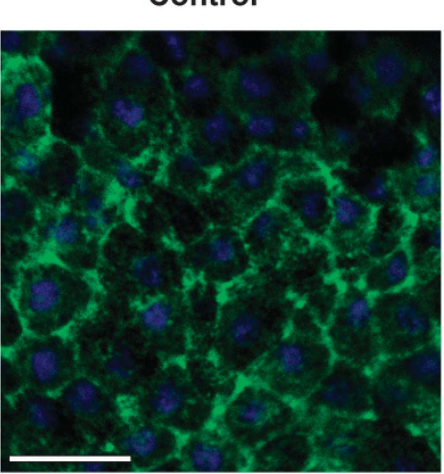

$\mathrm{xRIPK} 4 \mathrm{Mo}_{\mathrm{atg}}$

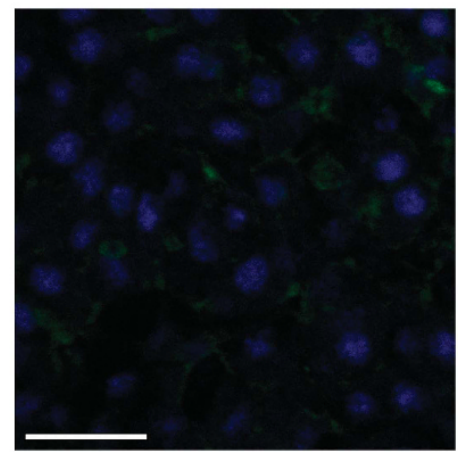

c

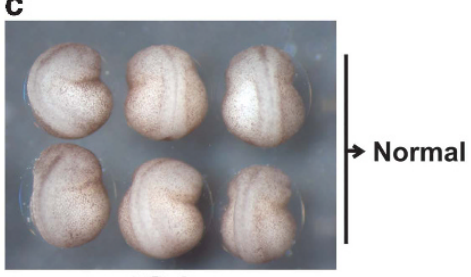

$\beta$ Gal

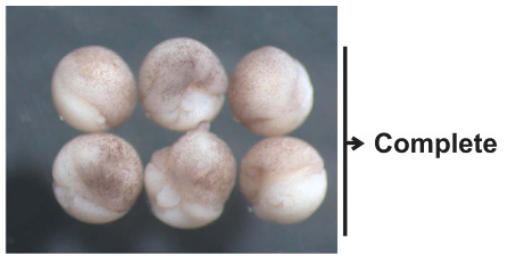

IRF6DBD

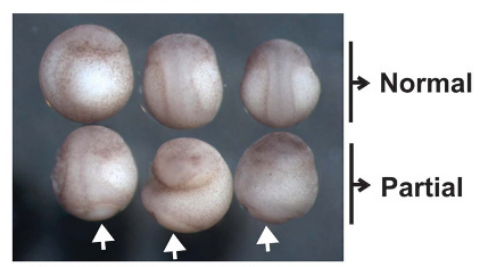

IRF6DBD + hRIPK4
IRF6DBD

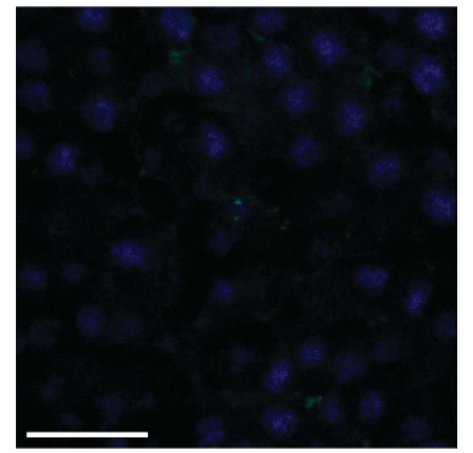

Figure 7 RIPK4 acts downstream of IRF6. (a) The RIPK4 promoter is activated by overexpressed IRF6. HEK293T cells were transfected with an hIRF6-expressing vector and a hRIPK4 promoter luciferase reporter construct. Luciferase activity was measured $24 \mathrm{~h}$ after transfection. Cumulative results of four independent experiments, each carried out in triplicate, are shown. Data show fold induction \pm s.d. Overexpression of Flag-tagged hIRF6 is shown by anti-Flag immunoblot. (b) Endogenous RIPK4 mRNA levels are reduced in embryos injected with dominant-negative IRF6. For xRIPK4 mRNA, Real-time quantitative PCR (RT-QPCR) was performed on 10 pooled $X$. tropicalis embryos injected with the indicated amounts of IRF6DBD synthetic mRNA. Error bars represent s.d. on triplicates. (c and d) Forced expression of hRIPK4WT rescues IRF6DBD-mediated gastrulation block. (c) Phenotypic analysis of Xenopus embryos injected with $500 \mathrm{pg}$ of IRF6DBD mRNA combined with $200 \mathrm{pg}$ of hRIPK4WTor $\beta$ Gal synthetic mRNA. The $\beta$ Galinjected embryos in Nieuwkoop stage 21 have completed gastrulation and neurulation (dorsal view, anterior to the top), while sibling IRF6DBD-injected embryos show complete exogastrulation (middle panel). Coinjection of hRIPK4WT rescues gastrulation in IRF6DBD-injected embryos completely or partially (lower panel). Arrows indicate minor exposures of endoderm, classifying those embryos as partially rescued. (d) Quantification of the experiment as shown in (c) summarizing the percentages of injected embryos with corresponding phenotypes. Total number of injected embryos is indicated above each bar as ' $n$ '. (e) Fluorescence microscopy analysis of total actin expression in ectodermal cells facing the animal blastocoel cavity of stage $11 X$. tropicalis embryos injected with the indicated constructs. Control $(n=14), \mathrm{XRIPK}_{\mathrm{M}} \mathrm{Mo}_{\text {atg }}(n=14)$ and IRF6DBD $(n=10)$. Both in XRIPK4Mo atg- and IRF6DBD-injected embryos a comparable depletion of cortical actin is observed in these BCR cells. Scale bars: $30 \mu \mathrm{m}$

\section{Discussion}

PPS are congenital disorders characterized by the presence of abnormal skin webbings (pterygia) behind the knee joint, often accompanied by other developmental defects affecting the skin, face and genitalia. The autosomal dominant form of PPS (MIM: 119500) is caused by mutations in the IRF6 gene, ${ }^{12,29}$ whereas the autosomal-recessive form of PPS, also referred to as BPS (MIM: 263650), was linked to inactivating
RIPK4 mutations. ${ }^{8,9}$ Despite the strong resemblance of these two syndromes, ${ }^{30}$ no molecular interconnection between IRF6 and RIPK4 was known.

Here, we describe a novel epistatic IRF6-RIPK4 signaling axis that links the phenotypes observed in both PPS syndromes. RIPK4 ${ }^{-1-}$ mice display abnormal apical localization of cadherin in several surface epithelia that show characteristics of aberrantly differentiated periderm, such as the oral cavity epithelia and skin. We believe that this ectopic 
cadherin localization can lead to increased adhesion and explain the fusion of apposed epithelia and the occurrence of cleft palate. The partial fusion of the limbs with the body surface in RIPK $4^{-1-}$ mice is in accordance with the epithelial fusions at flexural surfaces of knees and elbows in PPS patients. The observation that the mouth cavity of RIPK4 ${ }^{-1-}$ embryos is formed but fuses from E13.5 onwards suggests that most of the developmental abnormalities observed in RIPK4 ${ }^{-1-}$ mice and BPS are secondary defects resulting from epithelial fusions and are not due to aberrations in the developmental program as such.

We found that the presence of nuclei in the most superficial layers of RIPK4 ${ }^{-1-}$ skin is due to impaired periderm shedding, rather than merely parakeratosis as suggested before. ${ }^{3}$ The defect in periderm shedding at E18.5 could be a consequence of incomplete cornification and lack of proteasomal degradation of desmosomes. Indeed, intact desmosomes were observed within and between the cornified patches and

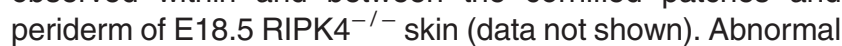
peridermal differentiation and adhesion has also been observed in mice mutant in IRF6 and Jagged2. ${ }^{31}$ Discontinuous cornified patches as in $\mathrm{RIPK}^{-1-}$ skin has not been reported for IRF6 ${ }^{-1-}$ mice, ${ }^{10}$ but was observed in $\mathrm{IRF6}^{+/ \mathrm{R}^{2} \mathrm{C}} \mathrm{SFN}^{+/ \mathrm{Er}}$ mice that are compound heterozygous for a disease-related IRF6 mutant and for a mutation in the phosphoserine-threonine-binding protein stratifin. ${ }^{11}$ This suggests that RIPK4 ${ }^{-1-}$ mice phenocopy mice with reduced but not complete loss of IRF6 activity, or that RIPK4 integrates signals from multiple developmental cues. As reported for IRF6 $^{-1-}$ keratinocytes, $^{13}$ we were unable to induce differentiation of cultured RIPK $4^{-1-}$ keratinocytes, which points to a cell autonomous defect. Partial keratinocyte differentiation in vivo, but in the absence of differentiation in vitro, was also observed for $\mathrm{IKK} 1^{-1-}$ skin. $^{32-35}$ However, the molecular mechanism explaining the difference between these in vivo and in vitro data remains elusive.

Although in Xenopus RIPK4 depletion by a splicing-blocking morpholino resulted in a late developmental phenotype partially reminiscent to what is observed in mice and humans, ${ }^{3,8,9}$ that is, cloacal fusion and anal atresia, the injection of a translation-blocking morpholino instead induced an early gastrulation defect, which phenocopies the developmental disruptions caused by interference with IRF6 function in both Xenopus and zebrafish. ${ }^{28}$ While splicing-blocking morpholinos only interfere with zygotic expression, translation-blocking morpholinos also target maternal transcripts, which could explain the observed phenotypic differences. Similar time-dependent differences in phenotype have been described for IRF6. ${ }^{10,11,28}$ Ectopic expression of WT hRIPK4 rescued the exogastrulation caused by IRF6DBD expression, suggesting that IRF6-dependent gastrulation in Xenopus is mediated, at least in part, through transcriptional regulation of RIPK4. Indeed, we found that IRF6 overexpression transactivated the hRIPK4 promoter. Blocking IRF6mediated transcription in frog embryos led to decreased RIPK4 mRNA levels. Moreover, IRF6 was also shown to be required for periderm differentiation and RIPK4 mRNA expression in zebrafish. ${ }^{36}$ The latter study also reports that the transcription factor Grhl3 (grainyhead-like 3) acts downstream of IRF6 to induce periderm differentiation in zebrafish.
However, Grh/3 mRNA expression levels were similar in WT and RIPK4 ${ }^{-1-}$ epidermis (Supplementary Figure S7). A very recent study found that RIPK4 can stimulate IRF6 transactivation and nuclear localization, probably by phosphorylating the IRF6 C-terminal domain. ${ }^{37}$ All these data suggest that mutual crosstalk between IRF6 and RIPK4 occurs in which IRF6 initially stimulates RIPK4 expression, which then further amplifies IRF6 activity. Besides IRF6, the transcription factor p63 was also reported to increase RIPK4 expression. ${ }^{9}$ However, we could not confirm p63-mediated transactivation of a RIPK4 promoter construct (data not shown). This could suggest that although the hRIPK4 genomic region contains p63-binding sites, the direct effect of p63 on RIPK4 expression is limited. In contrast, p63 might indirectly affect RIPK4 expression via its effect on IRF6 expression. ${ }^{38}$ This increase in IRF6 may then lead to p63 degradation in keratinocytes, thereby restricting the proliferative potential and promoting keratinocyte differentiation. ${ }^{39}$ Our data do not exclude that under certain conditions an interplay between IRF6 and p63 directly enhances RIPK4 promoter activity.

We found that all RIPK4 biological functions investigated are dependent on its kinase activity. Likewise, kinase-dead BPSassociated RIPK4 mutants could not rescue the gastrulation block caused by RIPK4 depletion. Consequently, overexpression of these mutants in HaCaT cells failed to induce scattering, except for the S376X mutant. The S376X mutant has an intact kinase domain and hence it could be a hypomorphic mutation rather than an amorphic one. This could explain why this mutant was identified in a non-lethal Bartsocas-Papas patient, ${ }^{8}$ whereas most of the patients die shortly after birth. Why this mutant does not rescue the exogastrulation phenotype in the complementation experiment in Xenopus embryos while it can still induce cell scattering and NF- $K B$ activation is not clear. It could be due to differences in sensitivity of the different assays used or to the fact that the S376X mutant lacks the ankyrin repeat domains, probably acting as a protein-protein interaction domain.

Although ectopic apical localization of E-cadherin could explain the epithelial fusions observed in RIPK $4^{-1-}$ mice and PPS patients, it is more difficult to clarify the gastrulation phenotype in RIPK4-depleted frog embryos. During gastrulation in Xenopus, involution of the mesoderm through the blastopore is coupled to epiboly movements where the BCR shows rapid thinning via radial intercalation of the deep animal ectodermal cells. ${ }^{26,27}$ This requires dynamic cadherinmediated interactions between the neighboring interdigitating cells. ${ }^{40}$ During this process, a decrease in C-cadherinmediated adhesiveness has been reported. ${ }^{41,42}$ Hence, the gastrulation block observed in Xenopus upon RIPK4 depletion can be due to the absence of dynamic cadherin activity, which may involve endocytosis and recycling of cadherin complexes and bidirectional interactions with the cytoskeleton. ${ }^{43}$ Indeed, in the RIPK4 morphants we observed only minor effects on cadherin expression levels, while the actin cytoskeleton was strongly affected. Moreover, membrane-bound cortical F-actin was also absent in epidermal cells of RIPK $4^{-1-}$ mice. Correct organization of the cortical actin cytoskeleton is essential for gastrulation movements in Xenopus. ${ }^{44,45}$ Our data suggest an important function for RIPK4 in regulating F-actin 
organization. How RIPK4 exactly affects the cytoskeleton and cellular adhesion remains to be determined.

Why RIPK4 or IRF6 depletion or inactivation results in a gastrulation defect in the frog but not in humans or mice may be due to the particular morphogenetic movements associated with gastrulation in Xenopus embryos. Compared with amniotes, in which gastrulation is more characterized by epithelial-to-mesenchymal transition-dependent cell ingression through the primitive streak, ${ }^{46}$ gastrulation in amphibian embryos involves the rapid invagination and involution of a circumferential band of mesodermal cells coupled to epiboly movements of the ectodermal animal pole cells. We believe that the disruption of these epiboly-associated movements due to the defective thinning of the animal BCR underlies the gastrulation defect in Xenopus. Gastrulation defects or cloacal swelling were not reported for RIPK4-depleted $X$. laevis embryos. ${ }^{22}$ However, it should be pointed out that in contrast to $X$. tropicalis (used in our study), $X$. laevis has two RIPK4 genes and the morpholinos used by Huang et al. ${ }^{22}$ contains mismatches with the $x$ Ripk4- $b$ gene, possibly explaining the absence of a gastrulation or cloacal phenotype in this study compared with ours. In line with the Huang et al. ${ }^{23}$ study, we found that RIPK4 depletion in $X$. tropicalis narrowed the expression domain of chordin, a dorsal organizer gene. This is consistent with a slight reduction in maternal $\mathrm{Wnt} / \beta$-catenin signaling. Because embryos depleted for maternal $\beta$-catenin do gastrulate normally, ${ }^{23}$ defective $\beta$-catenin signaling cannot explain the gastrulation defect caused by xRIPK4 depletion. Likewise, keratinocyte-specific $\beta$-catenin deletion did not result in epidermal and oral fusions. ${ }^{47}$ This suggests that in addition to Wnt/ $\beta$-catenin signaling, RIPK4 has to exert additional functions.

In summary, we identified a novel IRF6-RIPK4 pathway. In addition, our data underscore the importance of correct periderm differentiation during mouse embryonic development. Importantly, we add RIPK4 as a new player regulating cytoskeletal dynamics and restricting intercellular adhesion. This observation could also have important consequences for other diseases, such as cancer, in which it is important to maintain strong adhesion between the primary tumor cells to avoid metastasis. However, the mechanisms downstream of RIPK4 signaling remain to be determined. Finally, the extreme similarity between BPS patients and RIPK4 ${ }^{-/-}$mice makes these mice a good model system for studying PPS syndromes.

\footnotetext{
Materials and methods

Animals. C57BL/6 RIPK4 ${ }^{+/}$- mice were kindly provided by Dr. P Holland (Amgen Inc., Seattle, WA, USA). Mice were housed at the VIB Department for Molecular Biomedical Research in either specific pathogen-free or conventional animal facilities. RIPK4 ${ }^{-1-}$ embryos were derived from timed $\mathrm{RIPK}^{+/-}$ intercrosses followed by cesarean section at the indicated gestation times. All experiments were conducted according to institutional, national and European animal regulations.
}

Skin barrier assays. For toluidine blue assay, embryos were dehydrated by incubating them for 1 min each in 25, 50 and $75 \%$ methanol in PBS, followed by $100 \%$ methanol. Subsequently, they were rehydrated, washed in PBS and stained for $1 \mathrm{~min}$ in $0.0125 \%$ toluidine blue O (Fisher Scientific, Erembodegem, Belgium) in PBS. After destaining in PBS, embryos were photographed with a digital camera.
For lucifer yellow penetration assay, whole embryos were stained in $1 \mathrm{mM}$ Lucifer yellow in PBS (Fluka Chemical Co, Bornem, Belgium) and killed after $1 \mathrm{~h}$ of incubation. Frozen sections were prepared from back skin samples, counterstained with Hoechst dye and imaged on an Olympus BX61 (Olympus, Tokyo, Japan) fluorescence microscope. The inside-out skin barrier was analyzed by dermal injection of EZ-Link Sulfo-NHS-LC-Biotin (Pierce Chemical Co, Rockford, IL, USA) in PBS containing $1 \mathrm{mM} \mathrm{CaCl}_{2}$ was injected into the dermis in the dorsal part of the neck of E18.5 embryos. After incubation for 30 min, embryos were killed and the skin was fixed and embedded in paraffin.

Histology and immunocytochemistry. Killed embryos were fixed overnight in $4 \%$ PAF, dehydrated and embedded in paraffin. Sections of $5 \mu \mathrm{m}$ were processed and stained with hematoxylin and eosin. For immunohistochemistry, antigen retrieval was performed by boiling in $1 \mathrm{x}$ target retrieval solution (Dako REAL, Dako Belgium, Heverlee, Belgium) and detection was carried out with primary antibodies. Primary antibodies were detected with the HRP (DAB) Kit (Dako EnVision System, Glostrup, Denmark) or Alexa Fluor secondary antibodies. Histological images were taken on an Olympus BX51 (Olympus) brightfield microscope. Confocal images were taken with a Leica SP5, (Leica Microsystems Belgium, Diegem, Belgium), making use of a $\times 63,1.4$ NA oil immersion lens. In case of phalloidin staining, killed embryos were isolated and skin tissue was prepared by embedding skin in Neg-50 frozen section medium (cat. no. 6506; Microm-Prosan, Merelbeke, Belgium). Sections of $10 \mu \mathrm{m}$ were thawed, fixed in $4 \%$ PFA in PBS for 15 min and washed three times with PBS. Then, sections were treated with $0.2 \%$ Triton X-100 in PBS, three times for 5 min with $0.05 \%$ Triton $\mathrm{X}-100,1 \mathrm{~h}$ with $1 \% \mathrm{BSA}$ and $0.05 \%$ Triton $\mathrm{X}-100$. Finally, sections were stained by incubating for $1 \mathrm{~h}$ with Phalloidin-TRITC (Sigma-Aldrich, Bornem, Belgium), washed three times with $0.05 \%$ Trition X-100 in PBS, stained with Hoechst and mounted. Confocal images were captured with a Zeiss LSM780 confocal microscope (Zeiss, Zaventem, Belgium), The pinhole was set at 1 airy unit and $2 x$ line averaging was applied to reduce noise. Fifteen Z-sections were made with a $0.49 \mu \mathrm{m}$ z-spacing.

Immunofluorescence staining and imaging of Xenopus animal caps. For anti-actin staining in Xenopus embryos, animal caps were excised at stage 11 and fixed in $3.7 \%$ formaldehyde for $30 \mathrm{~min}$. Fixed caps were then washed in PBT (PBS + 0.1\% Tween-20), blocked with 10\% normal goat serum at room temperature for $1 \mathrm{~h}$ and then incubated with anti-actin monoclonal antibody (1: 1000; MP Biomedicals, Santa Ana, CA, USA) overnight at $4{ }^{\circ} \mathrm{C}$, followed by extensive washing with PBS, $0.1 \%$ Triton X-100. An Alexa Fluor 488-conjugated goat anti-mouse IgG (1:500; Life Technologies, Merelbeke, Belgium) was then added. All caps were imaged with the same imaging parameter setup.

Electron microscopy. For transmission electron microscopy, small fragments of E18.5 embryonic skin of WT and KO mice were submerged in a fixative solution of $2.5 \%$ glutaraldehyde and $3 \%$ formaldehyde in $0.1 \mathrm{M} \mathrm{Na}$-cacodylate buffer with $\mathrm{CaCl}_{2}(200 \mathrm{mg} / \mathrm{l})$, placed in a vacuum oven for $30 \mathrm{~min}$ and then left rotating for $3 \mathrm{~h}$ at room temperature. The solution was replaced with fresh fixative and samples were left rotating overnight at $4^{\circ} \mathrm{C}$.

After washing, samples were postfixed in $1 \% \mathrm{OsO}_{4}$ with $\mathrm{K}_{3} \mathrm{Fe}(\mathrm{CNN})_{6}$ in $0.1 \mathrm{M}$ $\mathrm{Na}$-cacodylate buffer, $\mathrm{pH}$ 7.2. Samples were dehydrated through a graded ethanol series, including staining with $2 \%$ uranyl acetate at the $50 \%$ ethanol step, and then embedded in Spurr's resin. Ultrathin sections of a gold interference color were cut with an ultramicrotome (Leica EM UC6, Leica Microsystems Belgium), Sections were imaged with a JEM 1010 (Jeol, Tokyo, Japan) transmission electron microscope.

Plasmids and lentiviral constructs. hRIPK4 and human E-cadherin cDNA were PCR amplified, with or without the stop codon (primer sequences; Supplementary Table S1), and cloned using CloneEZ (Genscript, Piscataway, NJ, USA) into pENTR3C (Invitrogen, Merelbeke, Belgium) digested with EcoRI and Xhol. RIPK4 mutants were generated by standard QuikChange mutagenesis (Stratagene, La Jolla, CA, USA) using pENTRhRIPK4 as template (primer sequences; Supplementary Table S1). All cDNAs were sequence verified at the pENTR3C level and transferred by Gateway LR-recombination into the appropriate destination vectors.

For Xenopus rescue experiments, LR-recombination reactions of stop codon bearing hRIPK4WT and mutants were performed with pdCS2-MT, constructed by rfA Gateway cassette insertion into pCS2-MT, which provides an N-terminal Myc-tag. zIRF6DBD was a kind gift from Dr. Robert A Cornell (University of lowa, lowa City, IA, USA). For inducible overexpression in $\mathrm{HaCaT}$ cells, LR-recombination reactions of 
stop codon-deleted variants were performed with modified versions of pLenti6/V5DEST (Invitrogen) that allow dox-inducible expression (cloning details available on request). A lentiviral NF- $\kappa B$ reporter construct (pGreenFire; SBI, Mountain View, CA, USA) was used to analyze RIPK4-induced NF- $\kappa$ B activity in HaCaT cells. A RIPK4 promoter construct (GeneCopoeia, Rockville, MD, USA; HPRM20329-PG02) was used to monitor RIPK4 promoter activity induced by IRF6.

Cell culture and lentiviral transduction. HEK293T and HaCaT cells were cultured in DMEM supplemented with $10 \%$ fetal calf serum, penicillin $(100 \mathrm{IU} / \mathrm{ml})$, streptomycin $(0.1 \mathrm{mg} / \mathrm{ml})$, L-glutamine $(0.03 \%)$ and sodium pyruvate $(0.4 \mathrm{mM})$. For lentivirus production, HEK293T cells were co-transfected with the modified pLenti6 plasmids and packaging plasmids (pMD2-VSV-G and pCMV delta R8,91). After $48 \mathrm{~h}$, the viral supernatant was harvested. HaCaT cells were transduced by two consecutive infections of $8 \mathrm{~h}$ each. After $48 \mathrm{~h}$, stably transduced cells were selected with puromycin, blasticidin or neomycin. After $24 \mathrm{~h}$, transgene expression was induced for the indicated time courses by the addition of $1 \mu \mathrm{g} / \mathrm{ml}$ dox (Sigma-Aldrich, Bornem, Belgium).

Reporter assays. For NF- $\kappa \mathrm{B}$ reporter assay, stable NF- $\kappa \mathrm{B}$ reporter (pGreenFire; SBI) containing HaCaT cells were transduced with inducible hRIPK4 constructs, as described in the previous section. Transgene expression was induced for $24 \mathrm{~h}$ with $1 \mu \mathrm{g} / \mathrm{ml}$ doxycycline; non-induced controls were included. Cells were lysed in luciferase lysis buffer ( $25 \mathrm{mM}$ Tris phosphate, pH 7.8, $2 \mathrm{mM}$ DTT, $2 \mathrm{mM}$ CDTA, 10\% glycerol and 1\% Triton X-100). Ten microliters of substrate buffer was added ( $658 \mu \mathrm{M}$ luciferin, $378 \mu \mathrm{M}$ coenzyme A and $742 \mu \mathrm{M}$ ATP) to $50 \mu \mathrm{l}$ lysate and luciferase activity (Luc) was assayed in a GloMax 96 Microplate Luminometer (Promega, Leiden, Netherlands). The obtained luciferase values were normalized to non-induced conditions. Equal amounts of lysates were analyzed by western blotting.

For measuring RIPK4 promoter activity, HEK293T cells were transfected with $25 \mathrm{ng}$ of RIPK4 promoter construct (GeneCopoeia; HPRM20329-PG02), $25 \mathrm{ng}$ of pACT $\beta$-galactosidase and $25 \mathrm{ng}$ of pcDNA hIRF6-Flag plasmid. Luciferase activity was assayed according to the manufacturer's instructions. Luc values were normalized for Gal values to correct for differences in transfection efficiency.

Primary keratinocyte isolation and differentiation. Primary keratinocytes were cultured in defined SKGM (Gibco, Life Technologies) supplemented with KGM Single Quots (Clonetics, San Diego, CA, USA). Embyros were dissected out and whole skin was isolated and disinfected in isobetadine for $30 \mathrm{~min}$. Skins were washed in $70 \%$ ethanol and gentamycin in PBS and incubated at $4{ }^{\circ} \mathrm{C}$ overnight while floating on $5 \mathrm{mg} / \mathrm{ml}$ dispase II in the keratinocyte medium. Epidermis was separated from the dermis and minced with a scalpel. The epidermal tissue pieces were then incubated in $0.5 \%$ trypsin/EDTA, with $1 \mu \mathrm{g} / \mathrm{ml}$ DNAsel (Roche, Vilvoorde, Belgium) for $10 \mathrm{~min}$ at $37^{\circ} \mathrm{C}$. For differentiation, cells were lysed with NP40 lysis buffer (20 mM Tris- $\mathrm{HCl}, \mathrm{pH}$ 8, $150 \mathrm{mM} \mathrm{NaCl}, 10 \%$ glycerol, 1\% NP40, 2 mM EDTA) after 3 days of postconfluent growth, in the keratinocyte medium or keratinocyte medium supplemented with $1.2 \mathrm{mM} \mathrm{Ca}^{2+}$ or $10^{-7} \mathrm{M} 1 \alpha, 25$-dihydroxide cholecalciferol (vitamin D; Sigma-Aldrich).

RNA/morpholino injections in Xenopus. $X$. tropicalis embryos were collected from natural mating couples and dejellied in $3 \%$ cysteine in $1 \times$ MMR buffer. Two-cell stage embryos were injected while submerged in $0.1 \times$ MMR containing $6 \%$ Ficol and cultured at $22{ }^{\circ} \mathrm{C}$. The morpholinos against XRIPK4 were designed by Gene Tool with nucleotide sequences: xRIPK4MOatg: $5^{\prime}$-ATGC GCCCTCCTTATCCATGGTGCC-3'; xRIPK4Mo E212: 5'-AGATATAACAGTCACTTA CCTTCAC-3'. As control, the standard control morpholino, CoMo (Gene Tool, Philomath, OR, USA) was used. Morpholino and CoMo were injected at the doses indicated in the figure legends. Capped RNA was synthesized by using SP6 RNA polymerase (Capped RNA Transcription Kit; Applied Biosystems, Life Technologies). For RNA extraction and quantitative RT-PCR, 10 embryos were pooled for each sample. Total RNA was then extracted using the Aurum Total RNA fatty and Fibrous Tissue Kit (Bio-Rad, Temse, Belgium). cDNA was synthesized from $1 \mu \mathrm{g}$ total RNA with oligo-dT and random hexamer primers using iScript cDNA Synthesis Kit (BioRad) according to the manufacturer's instruction. Real-time qPCR analysis was performed in triplicate using the qPCR Kit SYBR Green (Roche Applied Biosystems, Vilvoorde, Belgium) on the LightCycler 480 Real-time PCR system (Roche). All primers were designed using the Primer Express 1.0 software (Perkin-Elmer Applied Biosystems, Waltham, MA, USA) and are shown in Supplementary Table S2. For western blotting, 10 embyros were lysed per sample, using NP40 lysis buffer (20 mM Tris-HCl, pH 8; 150 mM NaCl; 10\% glycerol; 1\% NP40; 2 mM EDTA). Half of the lysate volume was then loaded on the SDS-PAGE gel.

Whole-mount in situ hybridization. Plasmid pPKS-Chordin (gift of Eric Bellefroid, ULB, Belgium) was linearized with EcoRI and a digitonin-Chordin antisense probe was synthesized using the T7 RNA polymerase and Digitonin Labeling Kit (Roche). Injected embryos were fixed in MEMFA (0.1 M MOPS, $2 \mathrm{mM}$ EGTA, $1 \mathrm{mM} \mathrm{MgSO}_{4}, 3.7 \%$ formaldehyde) and whole-mount in situ hybridization (WISH) was performed as essentially described in Sive et al. 2000 (Ref. 7 in Supplementary Information).

Western blotting. Equal amounts of lysates were separated by SDS-PAGE, transferred to nitrocellulose and analyzed by immunoblotting. Proteins were detected using the following antibodies: anti-Flag M2-HRP-conjugated antibody (Sigma-Aldrich), caspase-14 (in-house polyclonal), filaggrin (Covance, Brussels, Belgium), anti-myc HRP conjugated (Invitrogen), $\beta$-tubulin-HRP (Abcam, Cambridge, UK), Actin (MP Biomedicals).

More detailed information on Materials and Methods is available as Supplementary Information.

\section{Conflict of Interest}

The authors declare no conflict of interest.

Acknowledgements. We thank A Bredan for editing the manuscript, Amgen SA for the RIK4 ${ }^{-1}$ mice, $\mathrm{P}$ Coulombe for anti-K17 antibodies and Robert A Conell for the zIRF6DBD construct. This research has been supported by the Flanders Institute for Biotechnology (VIB); European Grants: FP6 Epistem LSHB-CT-2005-019067, FP7 TuMIC 2008-201662, COST SKINBAD BM0903 and Euregional PACT II; Belgian Grants: Interuniversity Attraction Poles, IAP 6/18 and IAP7/32; Stichting tegen Kanker 2010-162; Flemish Grants: FWO-Vlaanderen, 1.5.058.12N, G.0544.11, G.0226.09, 1.5.169.08N, G.0A45.12N, G.0214.09N, G.0942.10N; Ghent University Grants: MRP, GROUP-ID consortium, Concerted Research Actions (01G019A8-B8). PV holds a Methusalem Grant (BOF09/01M00709) from the Flemish Government. GB and BDC were supported by a Ghent University GOA. PDG, MF and CR obtained a PhD fellowship with the 'Instituut voor Innovatie door Wetenschap en Technologie'. SL is a postdoctoral fellow with the 'Fonds voor Wetenschappelijk Onderzoek-Vlaanderen'.

\section{Study Highlights}

- RIPK4 regulates keratinocyte differentiation and periderm maturation.

- RIPK4 deficiency in mice results in abnormal E-cadherin localization at the outer skin surface.

- RIPK4 can complement IRF6-dependent gastrulation defects in Xenopus.

- IRF6 controls RIPK4 expression.

1. Hoyes AD. Electron microscopy of the surface layer (periderm) of human foetal skin. J Anat 1968; 103(Part 2): 321-336.

2. Holbrook KA, Odland GF. The fine structure of developing human epidermis: light, scanning, and transmission electron microscopy of the periderm. J Invest Dermatol 1975; 65: 16-38.

3. Holland P, Willis C, Kanaly S, Glaccum M, Warren A, Charrier K et al. RIP4 is an ankyrin repeat-containing kinase essential for keratinocyte differentiation. Curr Biol 2002; 12 : 1424-1428.

4. Rountree RB, Willis CR, Dinh H, Blumberg H, Bailey K, Dean Jr C et al. RIP4 regulates epidermal differentiation and cutaneous inflammation. J Invest Dermatol 2010; 130: 102-112.

5. Adams S, Pankow S, Werner S, Munz B. Regulation of NF-kappaB activity and keratinocyte differentiation by the RIP4 protein: implications for cutaneous wound repair. $J$ Invest Dermatol 2007; 127: 538-544.

6. Meylan E, Tschopp J. The RIP kinases: crucial integrators of cellular stress. Trends Biochem Sci 2005; 30: 151-159.

7. Zhang D, Lin J, Han J. Receptor-interacting protein (RIP) kinase family. Cell Mol Immunol 2010; 7: 243-249

8. Mitchell K, O'Sullivan J, Missero C, Blair E, Richardson R, Anderson B et al. Exome sequence identifies RIPK4 as the Bartsocas-Papas syndrome locus. Am J Hum Genet 2012; 90: 69-75. 
9. Kalay E, Sezgin O, Chellappa V, Mutlu M, Morsy H, Kayserili H et al. Mutations in RIPK4 cause the autosomal-recessive form of popliteal pterygium syndrome. Am J Hum Genet 2012; 90: 76-85.

10. Ingraham CR, Kinoshita A, Kondo S, Yang B, Sajan S, Trout KJ et al. Abnormal skin, limb and craniofacial morphogenesis in mice deficient for interferon regulatory factor 6 (Irf6) Nat Genet 2006; 38: 1335-1340.

11. Richardson RJ, Dixon J, Malhotra S, Hardman MJ, Knowles L, Boot-Handford RP et al. Irf6 is a key determinant of the keratinocyte proliferation-differentiation switch. Nat Genet 2006; 38 : 1329-1334.

12. Kondo S, Schutte BC, Richardson RJ, Bjork BC, Knight AS, Watanabe $Y$ et al. Mutations in IRF6 cause Van der Woude and popliteal pterygium syndromes. Nat Genet 2002; 32: 285-289.

13. Biggs LC, Rhea L, Schutte BC, Dunnwald M. Interferon regulatory factor 6 is necessary, but not sufficient, for keratinocyte differentiation. J Invest Dermatol 2012; 132: 50-58.

14. Niessen CM, Leckband D, Yap AS. Tissue organization by cadherin adhesion molecules: dynamic molecular and cellular mechanisms of morphogenetic regulation. Physiol Rev2011; 91: 691-731.

15. McGowan KM, Coulombe PA. Onset of keratin 17 expression coincides with the definition of major epithelial lineages during skin development. J Cell Biol 1998; 143: 469-486.

16. Mazzalupo S, Coulombe PA. A reporter transgene based on a human keratin 6 gene promoter is specifically expressed in the periderm of mouse embryos. Mech Dev 2001; 100: 65-69.

17. Whittaker DK, Adams D. The surface layer of human foetal skin and oral mucosa: a study by scanning and transmission electron microscopy. J Anat 1971; 108(Part 3): 453-464.

18. Hardman MJ, Sisi P, Banbury DN, Byrne C. Patterned acquisition of skin barrier function during development. Development 1998; 125: 1541-1552.

19. Devin A, Cook A, Lin Y, Rodriguez Y, Kelliher M, Liu Z. The distinct roles of TRAF2 and RIP in IKK activation by TNF-R1: TRAF2 recruits IKK to TNF-R1 while RIP mediates IKK activation. Immunity 2000; 12: 419-429.

20. Thome M, Hofmann K, Burns K, Martinon F, Bodmer JL, Mattmann C et al. Identification of CARDIAK, a RIP-like kinase that associates with caspase-1. Curr Biol 1998; 8: 885-888.

21. Meylan E, Martinon F, Thome M, Gschwendt M, Tschopp J. RIP4 (DIK/PKK), a novel member of the RIP kinase family, activates NF-kappa $B$ and is processed during apoptosis. EMBO Rep 2002; 3: 1201-1208.

22. Huang X, McGann JC, Liu BY, Hannoush RN, Lill JR, Pham V et al. Phosphorylation of dishevelled by protein kinase RIPK4 regulates Wnt signaling. Science 2013; 339: 1441-1445.

23. Heasman J, Crawford A, Goldstone K, Garner-Hamrick P, Gumbiner B, McCrea P et al. Overexpression of cadherins and underexpression of beta-catenin inhibit dorsal mesoderm induction in early Xenopus embryos. Cell 1994; 79: 791-803.

24. Keller R. Shaping the vertebrate body plan by polarized embryonic cell movements. Science 2002; 298: 1950-1954.

25. Solnica-Krezel L. Conserved patterns of cell movements during vertebrate gastrulation. Curr Biol 2005; 15: R213-R228.

26. Keller R, Davidson LA, Shook DR. How we are shaped: the biomechanics of gastrulation. Differentiation 2003; 71: 171-205.

27. Keller RE. The cellular basis of epiboly: an SEM study of deep-cell rearrangement during gastrulation in Xenopus laevis. J Embryol Exp Morphol 1980; 60: 201-234.

28. Sabel JL, d'Alencon C, O'Brien EK, Van Otterloo E, Lutz K, Cuykendall TN et al. Maternal interferon regulatory factor 6 is required for the differentiation of primary superficial epithelia in Danio and Xenopus embryos. Dev Biol 2009; 325: 249-262.
29. Matsuzawa N, Kondo S, Shimozato K, Nagao T, Nakano M, Tsuda M et al. Two missense mutations of the IRF6 gene in two Japanese families with popliteal pterygium syndrome. Am J Med Genet A 2010; 152A: 2262-2267.

30. Bartsocas CS, Papas CV. Popliteal pterygium syndrome. Evidence for a severe autosomal recessive form. J Med Genet 1972; 9: 222-226.

31. Richardson RJ, Dixon J, Jiang R, Dixon MJ. Integration of IRF6 and Jagged2 signalling is essential for controlling palatal adhesion and fusion competence. Hum Mol Genet 2009; 18 : 2632-2642.

32. Hu Y, Baud V, Oga T, Kim KI, Yoshida K, Karin M. IKKalpha controls formation of the epidermis independently of NF-kappaB. Nature 2001; 410: 710-714.

33. Hu Y, Baud V, Delhase M, Zhang P, Deerinck T, Ellisman M et al. Abnormal morphogenesis but intact IKK activation in mice lacking the IKKalpha subunit of IkappaB kinase. Science 1999; 284: 316-320.

34. Takeda K, Takeuchi O, Tsujimura T, Itami S, Adachi O, Kawai T et al. Limb and skin abnormalities in mice lacking IKKalpha. Science 1999; 284: 313-316.

35. Gareus R, Huth M, Breiden B, Nenci A, Rosch N, Haase I et al. Normal epidermal differentiation but impaired skin-barrier formation upon keratinocyte-restricted IKK1 ablation. Nat Cell Biol 2007; 9: 461-469.

36. de la Garza G, Schleiffarth JR, Dunnwald M, Mankad A, Weirather JL, Bonde G et al. Interferon regulatory factor 6 promotes differentiation of the periderm by activating expression of grainyhead-like 3. J Invest Dermatol 2013; 133: 68-77.

37. Kwa MQ, Huynh J, Aw J, Zhang L, Nguyen T, Reynolds EC et al. Receptor-interacting protein kinase 4 and interferon regulatory factor 6 function as a signaling axis to regulate keratinocyte differentiation. J Biol Chem 2014; 289: 31077-31087.

38. Thomason HA, Zhou H, Kouwenhoven EN, Dotto GP, Restivo G, Nguyen BC et al Cooperation between the transcription factors p63 and IRF6 is essential to prevent cleft palate in mice. J Clin Invest 2010; 120: 1561-1569.

39. Moretti F, Marinari B, Lo lacono N, Botti E, Giunta A, Spallone G et al. A regulatory feedback loop involving p63 and IRF6 links the pathogenesis of 2 genetically different human ectodermal dysplasias. J Clin Invest 2010; 120: 1570-1577.

40. Winklbauer R. Cadherin function during Xenopus gastrulation. Sub-cell Biochem 2012; 60 301-320.

41. Brieher WM, Gumbiner BM. Regulation of C-cadherin function during activin induced morphogenesis of Xenopus animal caps. J Cell Biol 1994; 126: 519-527.

42. Gumbiner BM. Regulation of cadherin-mediated adhesion in morphogenesis. Nat Rev Mol Cell Biol 2005; 6: 622-634.

43. Collinet C, Lecuit T. Stability and dynamics of cell-cell junctions. Progr Mol Biol Transl Sci 2013; 116: 25-47.

44. Marsden M, DeSimone DW. Regulation of cell polarity, radial intercalation and epiboly in Xenopus: novel roles for integrin and fibronectin. Development 2001; 128: 3635-3647.

45. Petridou NI, Stylianou P, Skourides PA. A dominant-negative provides new insights into FAK regulation and function in early embryonic morphogenesis. Development 2013; 140: 4266-4276.

46. Arnold SJ, Robertson EJ. Making a commitment: cell lineage allocation and axis patterning in the early mouse embryo. Nat Rev Mol Cell Biol 2009; 10: 91-103.

47. Huelsken J, Vogel R, Erdmann B, Cotsarelis G, Birchmeier W. Beta-catenin controls hair follicle morphogenesis and stem cell differentiation in the skin. Cell 2001; 105: 533-545. 